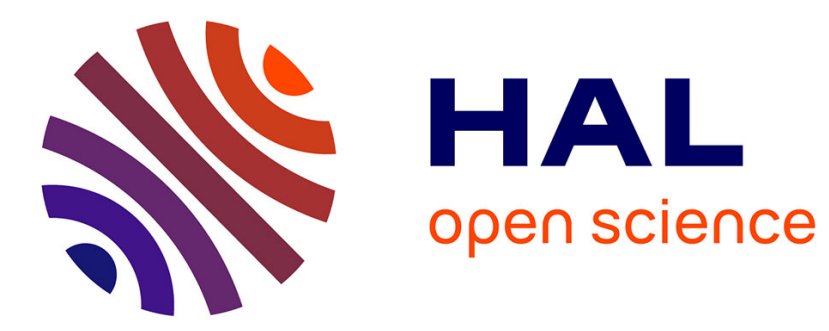

\title{
Perfusion deconvolution in DSC-MRI with dispersion-compliant bases
}

Marco Pizzolato, Timothé Boutelier, Rachid Deriche

\section{To cite this version:}

Marco Pizzolato, Timothé Boutelier, Rachid Deriche. Perfusion deconvolution in DSCMRI with dispersion-compliant bases. Medical Image Analysis, 2017, 36, pp.197 - 215. 10.1016/j.media.2016.12.001 . hal-01536292

\section{HAL Id: hal-01536292 \\ https://hal.inria.fr/hal-01536292}

Submitted on 10 Jun 2017

HAL is a multi-disciplinary open access archive for the deposit and dissemination of scientific research documents, whether they are published or not. The documents may come from teaching and research institutions in France or abroad, or from public or private research centers.
L'archive ouverte pluridisciplinaire HAL, est destinée au dépôt et à la diffusion de documents scientifiques de niveau recherche, publiés ou non, émanant des établissements d'enseignement et de recherche français ou étrangers, des laboratoires publics ou privés. 


\title{
Perfusion Deconvolution in DSC-MRI with Dispersion-Compliant Bases
}

\author{
Marco Pizzolato ${ }^{\mathrm{a}, *}$, Timothé Boutelier $^{\mathrm{b}}$, Rachid Deriche $^{\mathrm{a}, 1}$ \\ ${ }^{a}$ Université Côte d'Azur, Inria, France \\ ${ }^{b}$ Olea Medical, La Ciotat, France
}

\begin{abstract}
Perfusion imaging of the brain via Dynamic Susceptibility Contrast MRI (DSC-MRI) allows tissue perfusion characterization by recovering the tissue impulse response function and scalar parameters such as the cerebral blood flow $(C B F)$, blood volume $(C B V)$ and mean transit time $(M T T)$. However, the presence of bolus dispersion causes the data to reflect macrovascular properties, in addition to tissue perfusion. In this case, when performing deconvolution of the measured arterial and tissue concentration time-curves it is only possible to recover the effective, i.e. dispersed, response function and parameters. We introduce Dispersion-Compliant Bases (DCB) to represent the response function in the presence and absence of dispersion. We perform in silico and in vivo experiments, and show that DCB deconvolution outperforms oSVD and the state-of-the-art CPI+VTF techniques in the estimation of effective perfusion parameters, regardless of the presence and amount of dispersion. We also show that DCB deconvolution can be used as a pre-processing step to improve the estimation of dispersion-free parameters computed with CPI+VTF, which employs a model of the vascular transport function to characterize dispersion. Indeed, in silico results show a reduction of relative errors up to $50 \%$ for dispersion-free $C B F$ and MTT. Moreover, the DCB method recovers effective response functions that comply with healthy and pathological scenarios, and offers the advantage of making no assumptions about the presence, amount, and nature of dispersion.
\end{abstract}

Keywords: Perfusion, Deconvolution, Dispersion, Delay

\footnotetext{
* Corresponding author

Email address: marco.pizzolato@inria.fr, pizzolato.marco@gmail.com (Marco Pizzolato)

${ }^{1}$ Telephone Number: + 33-492387832
} 


\section{Introduction}

Perfusion imaging via Dynamic Susceptibility Contrast MRI (DSC-MRI) provides useful information in neurological diagnosis, characterization, and grading of brain tumors (such as gliomas), and ischemic strokes (Shiroishi et al., 2015). This information is supplied to physicians in the form of hemodynamic/perfusion parameters brain maps, such as the cerebral blood flow $(C B F)$, blood volume $(C B V)$ and mean transit time $(M T T)$. These maps are usually calculated, for each voxel, from the measured arterial and tissue concentration time-curves, $C_{a}(t)$ and $C_{t s}(t)$, which result from the intravenous injection of a tracer. The voxel-wise computation of $C B F$ and $M T T$ is closely related to the estimation of the time-dependent residual amount of tracer - the residue function ${ }^{2} r(t)$ - via deconvolution. In fact, the perfusion problem can be modeled as a linear system where $C_{a}(t)$ is the input, $C_{t s}(t)$ the output, and $R(t)=C B F \cdot r(t)$ is the unknown convolution kernel, i.e. the tissue impulse response function, which encodes capillary hemodynamic information describing the perfusion (Østergaard et al., 1999). However, in perfusion data processing the true arterial concentration is also unknown and can differ from the measured $C_{a}(t)$. Indeed, $C_{a}(t)$ is typically measured once in an arterial blood area such as in the carotid or middle cerebral artery (Rosen et al., 1991; Calamante et al., 1999) - but the bolus of tracer might undergo dispersion along the path to a voxel of interest, where the tissue concentration $C_{t s}(t)$ is measured. Dispersion is frequent in perfusion data especially of patients with cerebral ischemia or in the presence of a steno-occlusive disease in the artery (Calamante et al., 2003b), but it can be also present in healthy subjects (Østergaard et al., 1998; Calamante et al., 2000). When dispersion is present, the deconvolution of the measured $C_{a}(t)$ and $C_{t s}(t)$ leads to the estimation of an effective response function $R^{*}(t)$, whose shape reflects properties of both vasculature and tissue perfusion (Calamante et al., 2000, 2003b; Willats et al., 2006). If dispersion is not modeled, then the estimates of hemodynamic parameters are affected. In particular the presence of dispersion can lead to $M T T$ overestimation and $C B F$ underestimation (Calamante et al., 2000). An additional vasculature-related issue is the presence of a time-delay $\tau$ between the measured concentrations $C_{a}(t)$ and $C_{t s}(t)$, which further complicates the characterization of response function and dispersion.

Several techniques have been proposed to perform perfusion deconvolution. The solution for the response function can be sought by adopting the mono/bi-exponential

\footnotetext{
${ }^{2}$ In literature it is usually indicated with capital " $\mathrm{R}$ ", however we reserve this symbol for the response function.
} 


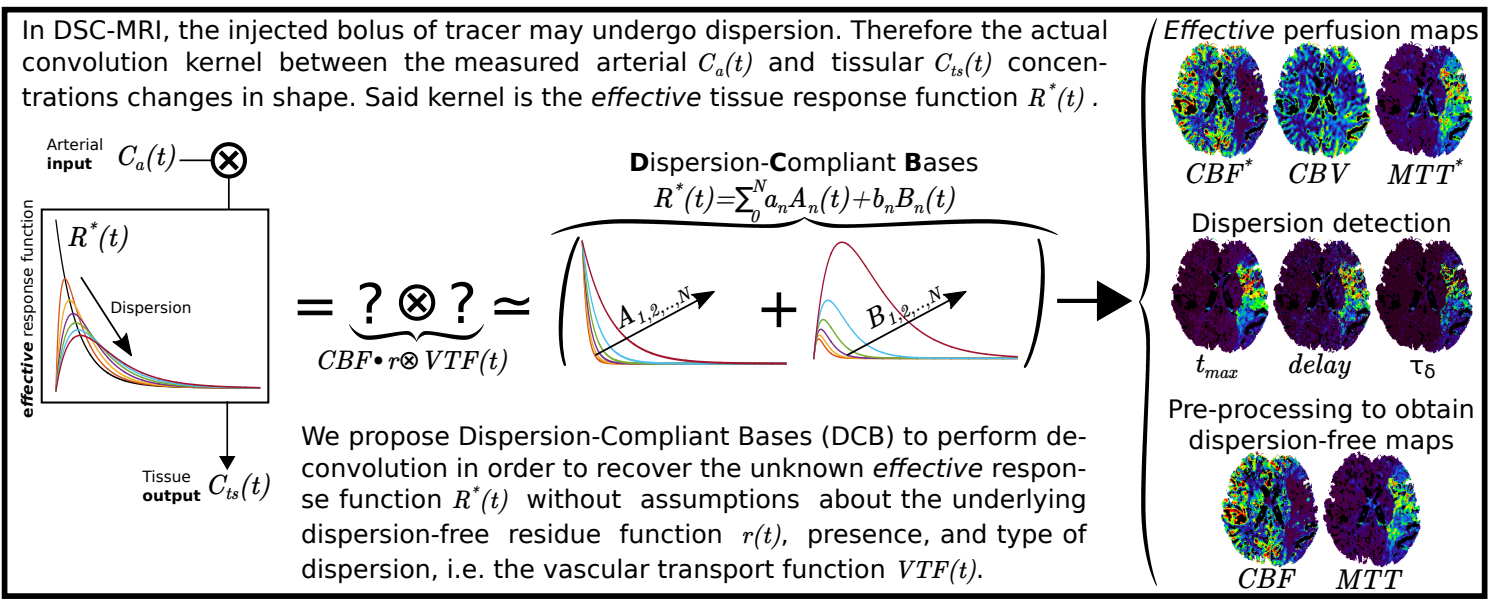

Figure 1: The Dispersion-Compliant Bases (DCB) to represent the unknown convolution kernel between the arterial and tissue concentration time-curves in the presence or absence of dispersion, and their usage to estimate effective perfusion parameters, performing dispersion detection, and pre-processing data to obtain dispersion-free parameters.

models from pharmacokinetics (Jacquez, 1972; Bassingthwaighte and Goresky, 1984; Lassen et al., 1988) or the vascular model (Mouridsen et al., 2006). Other approaches adopt the truncated singular value decomposition tSVD (Østergaard et al., 1996) and its variants (Wu et al., 2003; Smith et al., 2004; Bjørnerud and Emblem, 2010), Tikhonov regularization (Calamante et al., 2003a), non-linear stochastic regularization (Zanderigo et al., 2009), maximum entropy principle (Charter and Gull, 1987; Drabycz et al., 2005), maximum likelihood estimation (Vonken et al., 1999a), fully bayesian methods (Boutelier et al., 2012), wavelets (Wirestam and Ståhlberg, 2005; Connelly et al., 2006), exponential approximation (Keeling et al., 2009), ARMA modeling (Batchelor et al., 2010), and control point interpolation (Mehndiratta et al., 2013). Some of these approaches allow for time-delay estimation/insensitivity and/or for non-negativity of the estimated response function but do not account, at least explicitly, for dispersion.

Dispersion has been widely studied in literature (Calamante et al., 2000, 2002, 2003b, 2006; Calamante, 2005; Ko et al., 2007; Willats et al., 2012; Chappell et al., 2013). It can be characterized by a Vascular Transport Function (VTF): this is the probability density function $V T F(t)$ of the vascular transit times $t$. Some methods for detecting or minimizing dispersion effects (Willats et al., 2006, 2008), and correcting or characterizing them (Zanderigo et al., 2009; Mouannes-Srour et al., 2012; Mehndiratta et al., 2014a) have been proposed. However these methods employ heuristics or assume models for the VTF which can condition the estimation of the 
effective response function $R^{*}(t)$ via deconvolution, biasing results. Moreover, dispersion might be completely absent in a specific voxel-region; therefore, deconvolution should be performed with the least amount of assumptions about it.

In this work, we propose a framework to perform perfusion deconvolution to estimate the effective response function and hemodynamic parameters with and without dispersion. At the same time, we estimate the time-delay existing between the measured arterial and tissue concentrations. A graphical representation of our approach is presented in fig. 1. The method we propose is based on the non-parametric representation of the response function with newly introduced Dispersion-Compliant Bases (DCB). This representation allows us to obtain the effective convolution kernel between the measured $C_{a}(t)$ and $C_{t s}(t)$ without assumptions about the presence of dispersion or the shape of the VTF. After this, we characterize the eventual presence and amount of dispersion based on a shape parameter computed on the obtained response function: the dispersion time $\tau_{\delta}$. We also show that our approach can be used to improve results of techniques that quantify dispersion by employing a model for the VTF, such as the CPI+VTF technique (Mehndiratta et al., 2014a) which uses a gamma dispersion kernel for it. Particularly, we propose to fit the VTF model-based technique directly on the effective response function obtained with our DCB-based deconvolution. We test the performance of our approach at different dispersion levels and with different dispersion kernels. We validate our approach through in silico experiments comparing with oSVD (Wu et al., 2003), and the recently proposed CPI (Mehndiratta et al., 2013) and CPI+VTF (Mehndiratta et al., 2014a) methods. We also provide qualitative and quantitative results on stroke MRI data.

Our paper is structured as follows. In section 2, we review the theory of perfusion processing and provide a detailed explanation of the proposed deconvolution approach by means of Dispersion-Compliant Bases, and of the comparing techniques. Then, we discuss the implementation of the methods, the synthetic data generation and the MRI dataset in section 3. In sections 4 and 5, we present the performed experiments and related results, which we discuss in section 6 to finally provide our conclusion in section 7 .

\section{Theory}

In the following, we recapitulate the theoretical background of perfusion (section 2.1), and discuss the change in the tissue impulse response function in the presence of dispersion (section 2.2). We then show the influence of dispersion on the perfusion parameters, and propose to characterize its eventual presence and amount with the dispersion time (section 2.3). Afterwords, we introduce our Dispersion- 
Compliant Bases (DCB) to represent the effective response function in the presence or absence of dispersion (section 2.4).

\subsection{Perfusion Background}

The characterization in vivo of perfusion by means of DSC-MRI is performed by injecting a bolus of tracer, i.e. a paramagnetic agent (PA), in the subject's vascular system. This characterization is based on the tracer kinetics (Zierler, 1962, 1965; Axel, 1980) under the assumption that the tracer is intravascular and the blood brain barrier is intact. The passage of the PA in correspondence of small blood vessels or capillaries leads to phase dishomogenization of spins due to susceptibility effects (Villringer et al., 1988; Rosen et al., 1990; Weisskoff et al., 1994; Kiselev and Posse, 1999). This leads to the transverse relaxation time shortening $-T_{2}^{*}$ in case of Gradient-Echo sequence and $T_{2}$ with Spin-Echo (Speck et al., 2000; Kiselev, 2005) - causing the signal intensity to decrease. As the tracer clears out, the signal intensity increases again. The measured time-dependent signal $S(t)$ is related to the concentration time-curve $C(t)$ via

$$
S(t)=S_{0} \cdot e^{-\kappa \cdot C(t) \cdot T E}
$$

where $S_{0}$ is the baseline signal before tracer arrival, $T E$ is the echo-time, and $\kappa$ is the transverse relaxivity of the contrast agent (Rosen et al., 1990).

According to the indicator-dilution theory (Meier and Zierler, 1954), the measured concentration in a tissue voxel, $C_{t s}(t)$, is expressed as the convolution between the arterial input concentration to the voxel, $C_{a}(t)$, and the unknown tissue impulse response function $R(t)=C B F \cdot r(t)$

$$
C_{t s}(t)=\int_{0}^{t} C_{a}(\theta) R(t-\theta) d \theta .
$$

The blood volume $C B V$ is expressed as the relative amount of tracer in the voxel with respect to the arterial reference and is calculated as the ratio between the integrals of the tissue and arterial concentrations

$$
C B V=\frac{\int_{0}^{t} C_{t s}(t) d t}{\int_{0}^{t} C_{a}(t) d t}
$$

where, as for eq. (2), we omit the correction constant for the difference in hematocrit between large and small blood vessels of the brain (Rempp et al., 1994; Østergaard et al., 1996; Knutsson et al., 2010) and the tissue density. The mean transit time 
$(M T T)$ describes the average time it takes for a particle of tracer entering the vascular system, underlying a voxel, to leave it definitely. According to the central volume theorem (Stewart, 1894; Hamilton et al., 1932) it can be expressed as

$$
M T T=\frac{C B V}{C B F}
$$

thus, it can be calculated knowing the other parameters. In this work, in order to compare the performance of the tested techniques, we adopt the central volume theorem to compute the cerebral blood volume (Vonken et al., 1999b; Bjørnerud and Emblem, 2010)

$$
C B V=\int R(t) d t
$$

The goal in perfusion deconvolution is to estimate the residue function $r(t)$ and the perfusion parameters $C B F, C B V, M T T$. These can be calculated from the knowledge of $R(t)$ and the use of eqs. (4) and (5).

In fact, in the absence of dispersion, the $C B F$ can be estimated as the maximum value of $R(t)$. Indeed, the residue function $r(t)$ specifies the fraction of tracer remaining in the tissue underlying the voxel during time, following a bolus injection. As a consequence, the function has its theoretical maximum for $t=0$, i.e. $r(0)=1$, which implies $R(0)=C B F$. The residue function then decreases during time with the clearance of the tracer. A recent work (Mehndiratta et al., 2014b) shows that the shape of $r(t)$ is best described in vivo by a bi-exponential model, a finding in agreement with previous literature (Park and Payne, 2013). This model accounts for fast and slow flowing capillary components

$$
r_{b i-e x p}(t)=f \cdot e^{-\tau_{F} t}+(1-f) \cdot e^{-\tau_{S} t}
$$

where $\tau_{F}$ and $\tau_{S}$ are the fast and slow time-rates respectively, and $f$ specifies the relative weight of the fast component.

The estimation of the perfusion parameters depends on the quality of the recovered $R(t)$ from the measured $C_{a}(t)$ and $C_{t s}(t)$. However, this generally requires solving a deconvolution problem, originating from eq. (2), which is ill-posed. In addition, the measured $C_{t s}(t)$ can be physiologically delayed with respect to the selected arterial input $C_{a}(t)$, causing a time-delay issue. Finally, the presence of dispersion can severely affect the shape of the response function.

\subsection{Influence of dispersion: the effective response function}

The bolus of tracer may undergo dispersion along the way to the voxel of interest, where the tissue concentration $C_{t s}(t)$ is measured. This effect is mathematically described as a convolution between the measured arterial input $C_{a}(t)$ and the Vascular 
Table 1: Dispersion kernels and related shape parameters for the case of low, medium and high dispersion as given by Mehndiratta et al. (2014a).

\begin{tabular}{llll}
\hline & low & medium & high \\
\hline EDK & $\beta=1$ & $\beta=1 / 2$ & $\beta=1 / 4$ \\
LNDK & $\mu=-1, \sigma=1$ & $\mu=-0.15, \sigma=0.75$ & $\mu=0.59, \sigma=0.78$ \\
GDK & $p=1, s=2$ & $p=3, s=1$ & $p=5, s=0.5$ \\
\hline
\end{tabular}

Transport Function (Calamante et al., 2000). Therefore the effective arterial input $C_{a}^{*}(t)$ is

$$
C_{a}^{*}(t)=C_{a} \otimes V T F(t)
$$

and the resulting tissue concentration

$$
\begin{aligned}
C_{t s}(t) & =C B F \cdot\left[C_{a} \otimes V T F(t)\right] \otimes r(t) \\
& =C B F \cdot C_{a} \otimes[V T F \otimes r(t)](t) \\
& =C_{a} \otimes R^{*}(t)
\end{aligned}
$$

where $R^{*}(t)=R \otimes V T F(t)$ is the effective dispersed tissue response function, which corresponds to the original only in the absence of dispersion, i.e. $R^{*}(t)=R(t) \Longleftrightarrow$ $V T F(t)=\delta(t)$. In the presence of dispersion, $V T F(t)$ is the probability density function of the occurrence of the vascular transit time $t$. The shape of VTF is an expression of the vascular dynamic and is unknown. In the literature there are different models for it, called dispersion kernels (DK). These are the exponential, EDK (Calamante et al., 2000), the lognormal, LNDK (Willats et al., 2007; Calamante, 2013), and the gamma, GDK (Chappell et al., 2013),

$$
V T F(t)=\left\{\begin{aligned}
E D K(t, \beta) & =\beta e^{-\beta t} \\
L N D K(t, \mu, \sigma) & =\frac{1}{t \sigma \sqrt{2 \pi}} e^{-\frac{(\ln (t)-\mu)^{2}}{2 \sigma^{2}}} \\
G D K(t, p, s) & =\frac{s^{1+s p}}{\Gamma(1+s p)} t^{s p} e^{-s t}
\end{aligned}\right.
$$

where $\beta, \mu, \sigma, p, s$ are shape parameters. For each DK a vascular mean transit time $M T T_{v}$, from the site of the measurement of the arterial concentration $C_{a}(t)$ to the voxel of interest, can be computed (Calamante et al., 2000). In the case of EDK this simply corresponds to $M T T_{v}=1 / \beta$ : a low value of $\beta$ corresponds to a pronounced dispersion effect. Similarly, levels of dispersion can be encoded for the other DKs. Table 1 reports the shape parameters in case of low, medium and high dispersion as given by Mehndiratta et al. (2014a). In the presence of dispersion, data support the effective $R^{*}(t)$ instead of $R(t)$, therefore the estimation of $C B F$ and the other perfusion parameters is also affected. 


\subsection{Perfusion parameters in the presence of dispersion, and dispersion time}

In this section, we illustrate the effect of dispersion on the measured perfusion parameters, and propose to quantify it with the dispersion time.

We start by deriving the effective response function $R^{*}(t)$ for the bi-exponential model in eq. (6) according to eq. (8) when $\operatorname{VTF}(t)=E D K(t, \beta)$ in eq. (9)

$$
\begin{aligned}
R^{*}(t)= & \frac{-\beta}{\left(\beta-\tau_{F}\right)\left(\beta-\tau_{S}\right)} \\
& \cdot\left[\left(f\left(\tau_{F}-\tau_{S}\right)+\beta-\tau_{F}\right) e^{-\beta t}\right. \\
& +f\left(\tau_{S}-\beta\right) e^{-\tau_{F} t} \\
& \left.+\left(f\left(\beta-\tau_{F}\right)-\beta+\tau_{F}\right) e^{-\tau_{S} t}\right] \cdot C B F
\end{aligned}
$$

which is valid for $\beta>0$ and depends on the original mean transit time $M T T=$ $f / \tau_{F}+(1-f) / \tau_{S}$.

The influence of dispersion on the effective response function is shown in fig. 2, where curves are generated with eq. (6) - multiplied by $C B F$ - in the absence of dispersion (purely decaying function), and with eq. (10) in case of dispersion (nonmonotonic curves). The top image shows that dispersion effects, such as the decrease of the peak and the broadening of the shape, increase with the vascular mean transit time $M T T_{v}$, i.e. $1 / \beta$, in the direction of the arrow. The effective response function tends to a purely decaying curve for $M T T_{v} \rightarrow 0(\beta \rightarrow \infty)$. The bottom image shows that, for a specific value of $M T T_{v}>0$, the dispersion effects are enhanced when $M T T$ is high. The figure also illustrates $C B F$, i.e. the peak of the dispersion-free curve in the top image, the effective blood flow $C B F^{*}$, i.e. the peak of a dispersed curve, the time-delay $\tau$ between $C_{a}(t)$ and $C_{t s}(t)$, and the time-of-maximum $t_{\max }$. In these circumstances, the effective mean transit time takes into account $C B F^{*}$; eq. (4) then becomes $M T T^{*}=C B V / C B F^{*}$.

We note that the time between the beginning of the effective response function, at time $\tau$, and its peak, at time $t_{m a x}$, is sensitive to the aforementioned dispersion effects, i.e. the joint contributions of dispersion and mean transit time. Here we refer to that as the dispersion time $\tau_{\delta}$

$$
\tau_{\delta}=t_{\max }-\tau
$$

defining the time-to-peak of $R^{*}(t)$. A positive value of dispersion time $\left(\tau_{\delta}>0\right)$ reveals the presence of dispersion whereas a value equal to zero $\left(\tau_{\delta}=0\right)$ implies the absence of dispersion. We propose to detect and characterize dispersion effects by calculating the dispersion time $\tau_{\delta}$ directly on the effective response function. Since this parameter depends only on the recovered $R^{*}(t)$, its estimation is general and 


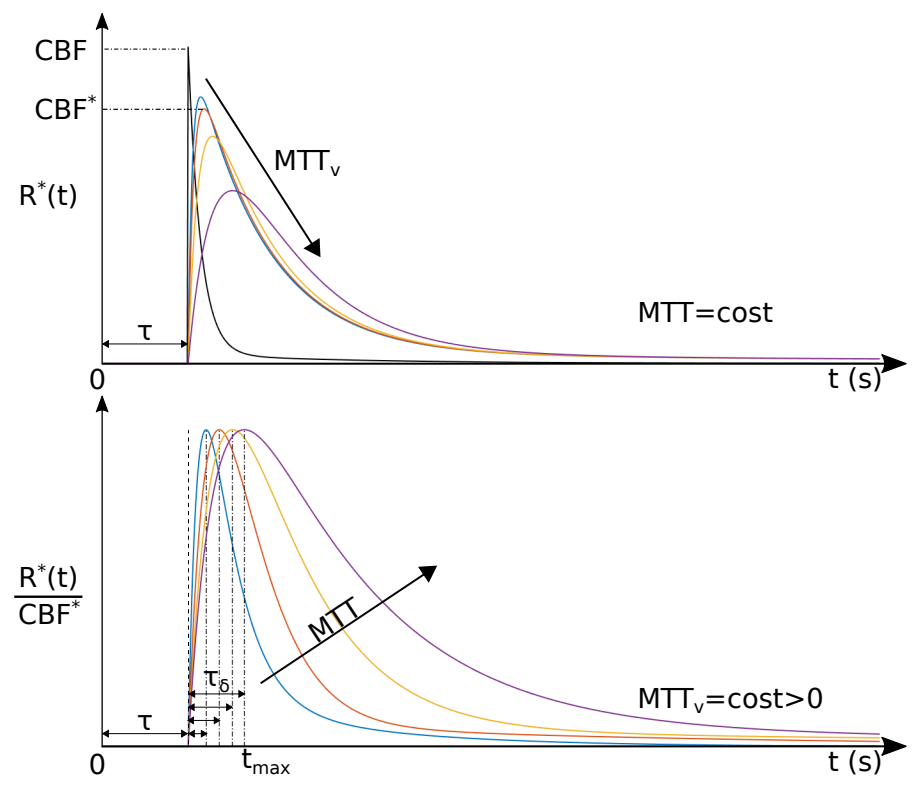

Figure 2: Influence of dispersion on the calculated effective response function, which includes $C B F$. Top: the maximum decreases $\left(C B F^{*}\right)$, and the time of the maximum $\left(t_{\max }\right)$ and dispersion time $\left(\tau_{\delta}\right)$ increase with the vascular mean transit time $M T T_{v}$. Bottom: $t_{\max }, \tau_{\delta}$ increase with $M T T$ (curves are normalized). The time-delay $\tau$ between $C_{t s}(t)$ and $C_{a}(t)$ determines the start of the curve.

does not assume a specific model for the underlying VTF. In the next section, we propose to perform deconvolution with bases that approximate the response function, even in the presence of dispersion, without making assumptions about the presence, amount or nature of dispersion itself.

\subsection{Dispersion-Compliant Bases}

In this section, we introduce the Dispersion-Compliant Bases (DCB) to represent the effective response function with or without dispersion. We propose to approximate $R^{*}(t)$ with a sum of $N$ bases where each basis is constituted as a sum of an exponential term and an exponential derivative term, with respect to the time rate, respectively weighted by two different constants $a_{n}$ and $b_{n}$. In order to let the bases render the most general solution we choose two different time rates $\alpha_{n}$ and $\beta_{n}$, respectively for the exponential and derivative terms. An illustration of the bases can be found in fig. 1. The sum of these bases can then represent any shape in the range between a multi-exponential decay and its derivative terms. The proposed 
approximation has the following expression

$$
R_{D C B}^{*}(t)=\Theta(t) \sum_{n=1}^{N}\left(a_{n} e^{-\alpha_{n} t}+b_{n} t e^{-\beta_{n} t}\right)
$$

where $\Theta(t)$ is the Heaviside step function with no half-maximum convention, i.e. $\Theta(t)=1 \forall t \geq 0$, and where the parameters $a_{n}, b_{n}, \alpha_{n}$ and $\beta_{n}$ are unknowns to be estimated. We note that the time-rates $\alpha_{n}, \beta_{n}$ enter non-linearly in the bases formulation. The time-delay $\tau$ between $C_{a}(t)$ and $C_{t s}(t)$ can be explicitly considered in the response function approximation because the convolution is commutative

$$
C_{a}(t-\tau) \otimes R^{*}(t)=C_{a}(t) \otimes R^{*}(t-\tau)
$$

therefore, when taking into account the time-delay eq. (12) becomes

$$
R_{D C B}^{*}(t)=\Theta(t-\tau) \sum_{n=1}^{N}\left[a_{n} e^{-\alpha_{n}(t-\tau)}+b_{n}(t-\tau) e^{-\beta_{n}(t-\tau)}\right]
$$

where we note that $\tau$ is an additional non-linear parameter to be estimated.

\subsubsection{Deconvolution with Dispersion-Compliant Bases}

The convolution problem in eq. (2) can be discretized by assuming that the tissue $C_{t s}(t)$ and the arterial $C_{a}(t)$ concentrations are measured on an equally spaced time grid $t_{1}, t_{2}, \ldots, t_{M}$ of size $M$, with $\Delta t=t_{i+1}-t_{i}$

$$
C_{t s}\left(t_{j}\right)=\Delta t \sum_{i=0}^{j} C_{a}\left(t_{i}\right) R^{*}\left(t_{j}-t_{i}\right)
$$

where we use $R^{*}(t)$ to underline the eventual presence of dispersion effects. The convolution in eq. (15) can be formulated in matrix form as $\mathbf{c}_{t s}=\mathbf{A r}$, where $\mathbf{A}$ is the $M \times M$ convolution matrix containing the samples of the arterial input concentration, $\mathbf{c}_{t s}$ contains the $M$ samples of the tissue concentration and $\mathbf{r}$ contains the $M$ unknown samples of the effective response function.

The unknown $M \times 1$ vector $\mathbf{r}$ is then computed via deconvolution. If a representation for the response function is defined, then the deconvolution problem aims to find the related set of unknown parameters. The representation in eq. (12) has linearly entering vector of coefficients $\mathbf{p}_{L}=\left[a_{1}, b_{1}, a_{2}, \ldots, a_{N}, b_{N}\right]$ and non-linearly entering vector of time-rates $\mathbf{p}_{N L}=\left[\alpha_{1}, \beta_{1}, \alpha_{2}, \ldots, \alpha_{N}, \beta_{N}\right]$, both of size $2 N \times 1$ with total number of unknowns $4 N \leq M$. Therefore the response function can be represented 
as $R^{*}(t)=g\left(t, \mathbf{p}_{L}, \mathbf{p}_{N L}\right)$. The convolution problem of eq. (15) can be reformulated in matrix form as

$$
\mathbf{c}_{t s}=\mathbf{A G}\left(\mathbf{p}_{N L}\right) \mathbf{p}_{L}
$$

where $\mathbf{G}\left(\mathbf{p}_{N L}\right)$ is the Dispersion-Compliant design matrix depending on $\mathbf{p}_{N L}$. Considering the maximum basis order $N$, the DCB design matrix and the corresponding vector of linear coefficients $\mathbf{p}_{L}$ are

$$
\begin{aligned}
\mathbf{G}\left(\mathbf{p}_{N L}\right) & =\left(\begin{array}{ccccc}
e^{-\alpha_{1} t_{1}} & t_{1} e^{-\beta_{1} t_{1}} & e^{-\alpha_{2} t_{1}} & \cdots & t_{1} e^{-\beta_{N} t_{1}} \\
e^{-\alpha_{1} t_{2}} & t_{2} e^{-\beta_{1} t_{2}} & e^{-\alpha_{2} t_{2}} & \cdots & t_{2} e^{-\beta_{N} t_{2}} \\
\vdots & \vdots & \vdots & \cdots & \vdots \\
e^{-\alpha_{1} t_{M}} & t_{M} e^{-\beta_{1} t_{M}} & e^{-\alpha_{2} t_{M}} & \cdots & t_{M} e^{-\beta_{N} t_{M}}
\end{array}\right) \\
\mathbf{p}_{L} & =\left(a_{1}, b_{1}, a_{2}, \ldots, b_{N}\right)^{T}
\end{aligned}
$$

where the matrix has dimension $M \times 2 N$, and the vector $2 N \times 1$.

The deconvolution problem incorporating eq. (12) can be solved as

$$
\hat{\mathbf{p}}_{L}, \hat{\mathbf{p}}_{N L}=\underset{\mathbf{p}_{L}, \mathbf{p}_{N L}}{\operatorname{argmin}}\left\|\mathbf{c}_{t s}-\mathbf{A G}\left(\mathbf{p}_{N L}\right) \mathbf{p}_{L}\right\|_{2}
$$

using a gradient-descent method (see section 3.2 for more details). The deconvolution problem incorporating eq. (14) includes the time-delay as additional non-linear parameter to be estimated. The time-delay $\tau$ is taken into account by considering the circular formulation of the convolution as for the case of cSVD (Wu et al., 2003). The measured arterial and tissue concentration time-curves of length $M$ are extended by zero-padding up to a length $L \geq 2 M$ to avoid aliasing. The circular $L \times L$ convolution matrix $\mathbf{A}^{c}$ has then entries

$$
A_{i, j}^{c}=\left\{\begin{array}{rl}
\Delta t \cdot C_{a}\left(t_{i-j+1}\right) & \text { for } j \leq i \\
\Delta t \cdot C_{a}\left(t_{L+i-j+1}\right) & \text { for } j>i
\end{array} .\right.
$$

We note that other formulations of the circular convolution matrix can be adopted. In general, higher order quadratures (trapezoidal or Simpson's integration rules) may provide more accurate approximations. We reformulate the convolution problem as $\mathbf{c}_{t s}=\mathbf{A}^{c} \mathbf{G}^{\tau}\left(\mathbf{p}_{N L}\right) \mathbf{p}_{L}$, where $\mathbf{G}^{\tau}\left(\mathbf{p}_{N L}\right)$ is the $L \times 2 N$ design matrix depending on $\tau$ and $\mathbf{p}_{N L}$ extended to the circular time sampling grid. We perform the estimation of $\tau$ via grid search over a range $\left[\tau_{\min }, \tau_{\max }\right]$ seconds (chosen by the user), with a time step $\tau_{s} \leq \Delta t$. The estimated vectors of parameters $\hat{\mathbf{p}}_{L}, \hat{\mathbf{p}}_{N L}$ can be again obtained via gradient-descent methods when the estimated delay $\hat{\tau}$ is that minimizing $\| \mathbf{c}_{t s}-$ $\mathbf{A}^{c} \mathbf{G}^{\tau}\left(\hat{\mathbf{p}}_{N L}\right) \hat{\mathbf{p}}_{L} \|_{2}$ among all $\tau \in\left[\tau_{\min }, \tau_{\max }\right]$. The use of functional bases like DCB offers the advantage of computing the analytic Jacobian matrix that can be supplied to the non-linear routine speeding up the estimation of the parameters. More details about the implementation of DCB deconvolution are provided in section 3.2. 


\section{Methods and Materials}

In this section, we present the techniques used for comparison with DCB (section 3.1) and the details about the implementation (section 3.2). We then explain the synthetic data generation (section 3.3) and provide details on the MRI dataset (section 3.4).

\subsection{Comparing techniques}

We compare our method with two techniques that are able to recover the effective response function in the presence of dispersion. These are the widely used blockcirculant singular value decomposition, (Wu et al., 2003) and the recent state-of-theart CPI+VTF method (Mehndiratta et al., 2014a).

The technique based on singular value decomposition uses the discretized version of the convolution in eq. (2) and calculate the pseudoinverse of the convolution matrix via SVD. This normally produces oscillations in the solution, due to noise, which are reduced using a threshold on the singular values (Østergaard et al., 1996). Among the several SVD versions proposed in literature, we adopt oSVD which iteratively seeks a solution that renders an oscillations index below a specified threshold Wu et al. (2003). The oSVD approach offers the advantage of being insensitive to time-delay and recovers the effective response function in the presence of dispersion (Mehndiratta et al., 2014a).

The CPI+VTF deconvolution (Mehndiratta et al., 2014a) is a method based on control point interpolation that overcomes the limitation of its ancestor CPI (Mehndiratta et al., 2013) by allowing the estimation of the effective response function in case of dispersion. The CPI technique uses consecutive control points opportunely constrained to represent the non-dispersed response function. Its CPI+VTF evolution uses this representation and convolves it with the gamma dispersion kernel (GDK) in eq. (9), which is the model selected for the VTF, allowing the estimation of the dispersion-free $C B F$ and $M T T$. This method revealed to be more effective than other approaches in the presence of dispersion (Mehndiratta et al., 2014a). Particularly the effectiveness of CPI+VTF was proved in synthetic experiments where the ground-truth dispersion kernel was the GDK, EDK or LNDK. On the other hand, CPI performs better on dispersion-free data than CPI+VTF (Mehndiratta et al., 2014a). For this reason, we adopt CPI instead of CPI+VTF when performing synthetic experiments in the absence of dispersion (see section 4.1).

\subsection{Implementation}

The oSVD and CPI+VTF techniques were set up following indications as given respectively in Wu et al. (2003) and Mehndiratta et al. (2014a). 
For oSVD, we point out that in this work, as indicated in Wu et al. (2003), the oscillations index threshold was found as the one minimizing the estimation error of $C B F^{*}$ on the entire generated synthetic dataset, which comprises all the tested $C B F, M T T$, delays, dispersion kernels and levels. For this reason, in the following in silico experiments oSVD will often show the best performance in $C B F^{*}$ estimates.

The CPI and CPI+VTF techniques rely on a previous mono-exponential nonlinear deconvolution to identify the initial guess for the subsequent non-linear routine (Mehndiratta et al., 2013, 2014a). In this work, we used CBF and MTT estimates found with oSVD as initial guess for the mono-exponential deconvolution.

For DCB, the maximum basis order was chosen after performing some synthetic experiments to determine $N$ such that it renders a good compromise between explaining the variety in the data, i.e. the different dispersion kernels (exponential, gamma, and lognormal), and robustness to noise. The idea is to find the minimum order that allows us to minimize the estimation error of the peak of the effective residue function, i.e. $C B F^{*}$, in the presence of noise. To do so, we synthetically generated effective response functions $R^{*}(t)$ obtained by convolving the bi-exponential $r(t)$ of eq. (6) with each of the various dispersion kernels: gamma (GDK), exponential (EDK), and lognormal (LNDK). For the bi-exponential $r(t)$, we chose the median normal tissue parameters given by Mehndiratta et al. (2014b): $f=0.97$, $\tau_{F}=0.68$ and $\tau_{S}=0.05$. For the dispersion kernel parameters we adopted the values corresponding to low, medium, and high dispersion (table 1). Secondly, for each generated $R^{*}(t)$ - resulting from the combination of dispersion kernel and intensity we performed 100 DCB fittings with each order $N \in\{2,3,4,5,6,7,8\}$ corresponding to as many noisy realizations in the case of high noise regime, with $S N R=10$. The order $N=5$ was found to be the minimum order reducing the $C B F^{*}$ estimation error below threshold. The initial guess for DCB parameters was based on oSVD results to approximate a mono-exponential decay, particularly $\mathbf{p}_{L}=\left[C B F_{o S V D}, 0, \ldots, 0\right]$ and $\alpha_{n}, \beta_{n}=1 / M T T_{o S V D} \forall n \in[1, N]$. To obtain the dispersion-free perfusion parameters $C B F$ and $M T T$ from the DCB solution, a CPI+VTF model fitting was performed on the effective dispersed response function. This model has been preferred to others to allow a homologous comparison between techniques.

The estimation of the parameters for $\mathrm{CPI}+\mathrm{VTF}$ and $\mathrm{DCB}$ deconvolutions was solved considering the minimization of the $l_{2}$ reconstruction norm with respect to the tissue concentration $C_{t s}(t)$. The techniques were implemented using in-house software written in $\mathrm{MATLAB}^{3}$, and the non-linear optimization was performed using the trust-region-reflective algorithm within the lsqnonlin function, which improves per-

\footnotetext{
${ }^{3}$ MATLAB Release 2015a, The MathWorks, Inc., Natick, Massachusetts, United States.
} 


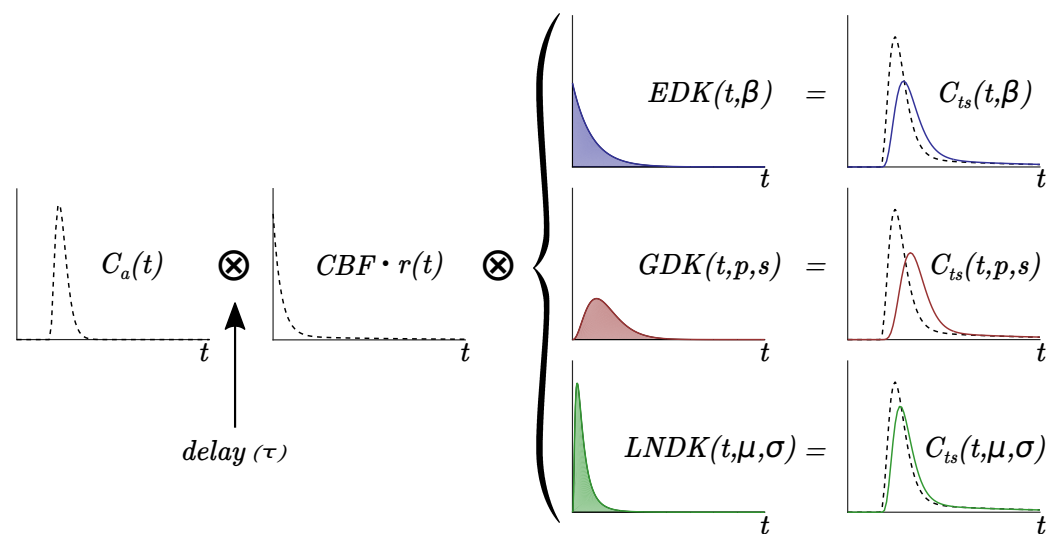

Figure 3: The relationship between the arterial input $C_{a}(t)$ and the tissue concentration $C_{t s}(t)$. Dashed lines indicate the non-dispersed case, i.e. the convolution kernel (response function) is only represented by the product $C B F \cdot r(t)$. Blue, red and green colors indicate the effect of the exponential (EDK), gamma (GDK) and lognormal (LNDK) dispersion kernels respectively, which are defined according to eq. (9). The presence of the time-delay $\tau$ is indicated with an arrow.

formance over Levenberg-Marquardt in case the initial guess is far from the solution.

\subsection{In silico data}

In this section, we describe the simulation of the MR arterial and tissue signals and concentrations used for the experiments in silico. The signals are obtained from the simulated concentration curves when $C B F, C B V, M T T$, time-delay $\tau$, dispersion kernel (GDK,EDK,LNDK), and dispersion level (none, low, medium, high as specified in table 1) are set. An illustration of the relationship between the generated arterial and tissue concentrations is shown in fig. 3 .

The arterial concentration is calculated as the gamma-variate function (Starmer and Clark, 1970)

$$
C_{a}(t)=\left\{\begin{aligned}
0 & \text { for } t \leq t_{0} \\
\gamma_{0}\left(t-t_{0}\right)^{\nu} \cdot e^{-\left(t-t_{0}\right) / \xi} & \text { for } t>t_{0}
\end{aligned}\right.
$$

with corresponding parameters - reported in table 2 - set according to the findings in Meijs et al. (2015). The tissue concentration $C_{t s}(t)$ is obtained by convolution between the arterial concentration $C_{a}(t)$ with the effective response function $R^{*}(t)$, according to eq. (15). This corresponds to the bi-exponential model (multiplied by $C B F)$ in eq. (6), or to its convolution with the selected dispersion kernel at the selected dispersion level. The parameters $f, \tau_{F}, \tau_{S}$ of the residue function in eq. (6) were set according to the optimal median values given by Mehndiratta et al. (2014b). 
The time-rates $\tau_{F}, \tau_{S}$ were varied together in percentage to render the desired value of $M T T=f / \tau_{F}+(1-f) / \tau_{S}$. The concentration time-curves are then converted into the corresponding signal intensities via eq. (1). The transverse relaxivity $\kappa$ is fixed according to Østergaard et al. (1996). For the arterial and tissue signals, the pre-injection baseline signal $S_{0}$ and the echo-time $T E$ are chosen according to Wirestam and Ståhlberg (2005). Signals are obtained to simulate a repetition time $T R=1 s$ and corrupted by Gaussian noise with zero mean and standard deviation $\sigma=S_{0} / S N R$ before being reconverted into concentration time-curves. Table 2 reports the values of the parameters used for the experiments. Presented results are obtained for $S N R=50$ (Boutelier et al., 2012; Mehndiratta et al., 2014a), which corresponds to an effective baseline SNR on the tissue signal around 16.7.

Table 2: Parameters used for in silico experiments.

\begin{tabular}{llllllllll}
\hline$t_{0}$ & $\gamma_{0}$ & $\nu$ & $\xi$ & $f$ & $(\kappa \cdot T E)^{a}$ & $S_{0}^{a}$ & $(\kappa \cdot T E)^{t s}$ & $S_{0}^{t s}$ & $T R$ \\
\hline $10 s$ & 1 & 3.66 & 1.8 & 0.97 & 0.1123 & 600 & 0.4751 & 200 & $1 s$ \\
\hline
\end{tabular}

\subsection{In vivo data}

The MR perfusion data was acquired from a 65-years-old female with left M1 distal occlusion three hours after onset. A total of $10 \mathrm{ml}$ of Gadolinium was administrated with rate $3 \mathrm{ml} / \mathrm{s}$, with $20 \mathrm{ml}$ of saline chase at the same speed. A $256 \times 256 \times 15$ volume with 60 time samples was acquired with a $1.5 T$ Siemens device using a SpinEcho sequence with $T E=52 \mathrm{~ms}$ and $T R=1.5 \mathrm{~s}$. The ratio between arterial and tissue transverse relaxivities is $\kappa_{\text {arterial }} / \kappa_{\text {tissue }}=0.04$ (Boutelier et al., 2012; Rohrer et al., 2005).

\section{In Silico Experiments and Results}

In this section, we present the ensemble of experiments performed to validate our method based on DCB deconvolution and the related results. We first present a comparison in the absence of dispersion between DCB, oSVD, and the state-of-theart CPI (section 4.1). Secondly, we study the influence of dispersion on the results obtained with non-parametric methods, particularly showing the drawbacks of oSVD in such a case (section 4.2). We then perform an extensive comparison of DCB with oSVD and CPI+VTF deconvolutions including all the dispersion kernels and levels (section 4.3). Finally, as an application of DCB deconvolution, we use it as a preprocessing step to improve the dispersion-free estimates of $C B F$ and $M T T$ obtained with CPI+VTF model fitting (section 4.4). 

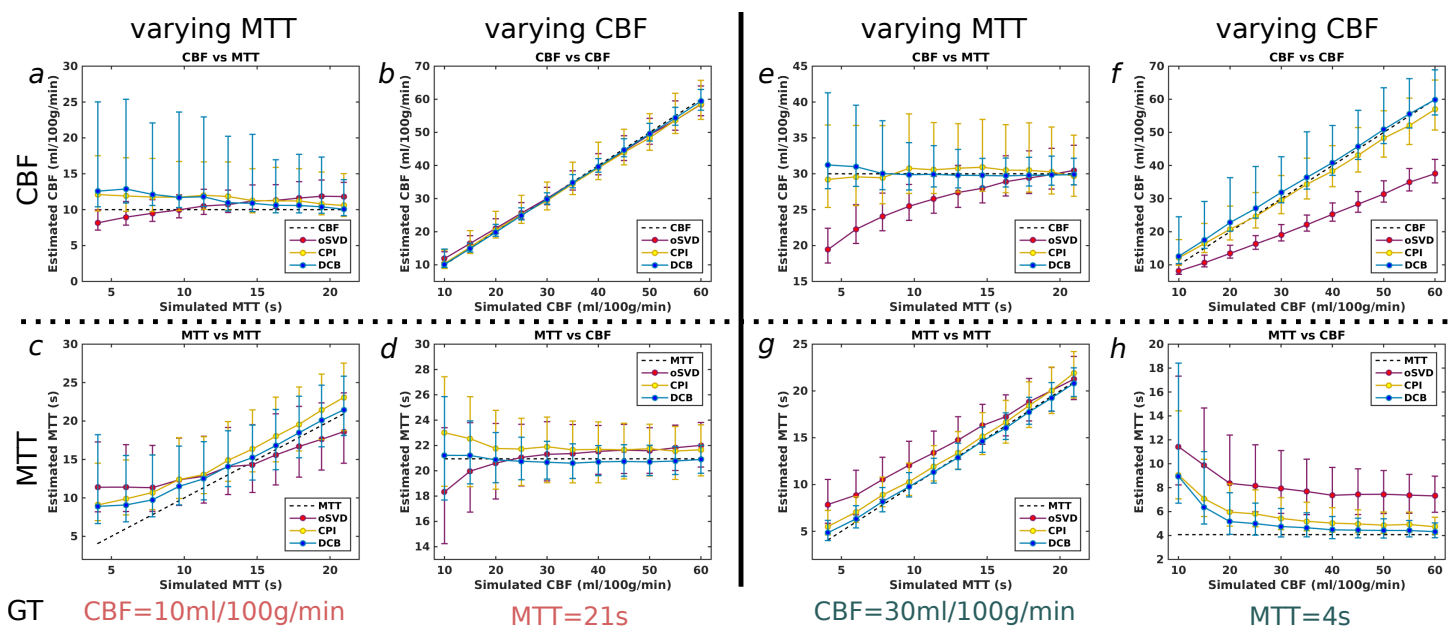

GT $\quad \mathrm{CBF}=10 \mathrm{ml} / 100 \mathrm{~g} / \mathrm{min}$

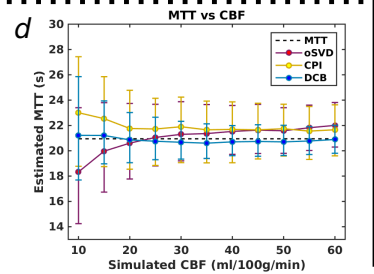

$\mathrm{MTT}=21 \mathrm{~s}$

$\mathrm{MTT}=4 \mathrm{~s}$

Figure 4: Dispersion-free comparison of $C B F$ (first row, $a, b, e, f$ ) and $M T T$ (second row, $c, d, g, h$ ) estimates, obtained with oSVD (purple), CPI (yellow) and DCB (blue). Data-point correspond to the average over 1000 repetitions, and bars indicate above and below average standard deviations. In each column, one of the ground-truth parameters $(M T T$ or $C B F)$ is varied according to the graphs' x-axis, while the other $(C B F$ or $M T T)$ is kept constant to the value indicated in the bottom under the tag "GT". On the left $(a, b, c, d)$, the GT values correspond to a low cerebral blood flow $(C B F=10 \mathrm{ml} / 100 \mathrm{~g} / \mathrm{min})$ and high mean transit time $(M T T \approx 21 \mathrm{~s})$ regime. A pathological scenario can be identified for low $C B F$ and high $M T T$, i.e. the right side of images $a, c$ and the left side of images $b, d$. In this condition, DCB shows the best attachment to the ground-truth (dashed lines). On the right $(e, f, g, h)$, the $\mathrm{GT}$ values correspond to a normal scenario $(C B F=30$ $\mathrm{ml} / 100 \mathrm{~g} / \mathrm{min}$ and $M T T=4 s)$. DCB results remain closer to the ground-truth. Moreover, DCB deconvolution reduces the standard deviation of $C B F$ estimates as $M T T$ increases $(e)$ and reduces the overestimation of $M T T$ at any $C B F(h)$.

\subsection{Dispersion-free comparison with oSVD and CPI}

In this section, we compare DCB with oSVD and CPI deconvolution methods in the absence of dispersion and delay. We compare with CPI because it is designed for dispersion-free data and performs better than its dispersion-compliant evolution CPI+VTF (Mehndiratta et al., 2014a). In fig. 4 we compare $C B F$ (first row) and $M T T$ (second row) estimates for two scenarios. In the left images $(a, b, c, d)$, the ground-truth values ("GT" tag in the bottom) are fixed to render a low cerebral blood flow $(C B F=10 \mathrm{ml} / 100 \mathrm{~g} / \mathrm{min}$ in $a, c)$ and high mean transit time $(M T T=21 \mathrm{~s}$ in $b, d)$ regime. In the right images $(e, f, g, h)$ the GT values $(C B F=30 \mathrm{ml} / 100 \mathrm{~g} / \mathrm{min}$ in $e, g$ and $M T T=4 s$ in $f, h)$ comply with a normal tissue scenario. On top of each column it is reported the perfusion parameter that is varied in the x-axis. A summary of the results follows. 


\subsubsection{Results}

The results in fig. 4 show that DCB (blue) and CPI (yellow) perform overall better than oSVD (purple). For instance, results in normal tissue scenario obtained with oSVD confirm the well-known $C B F$ underestimation $(e, f)$ and $M T T$ overestimation $(g, h)$. However when the actual SNR is low, oSVD (also aided by the optimal setting described in section 3.2) helps the estimation. Indeed, in the left side of the image (a) oSVD renders the best estimates of $C B F$, whereas DCB has the highest standard deviation. This is due to the very low effective signal-to-noise ratio - low $C B F$, low $M T T$ and SNR $=50-$ which penalizes the higher flexibility of DCB compared to CPI and to the robustness to noise of oSVD. On the other hand, oSVD has the worst performance in MTT estimation.

In general, CPI and DCB have similar performance. However, in normal tissue scenario DCB reduces the standard deviation in $C B F$ estimation as $M T T$ increases (e), and shows the best recovery of $M T T$ as the $C B F$ increases $(h)$. Moreover, in the low $C B F$ and high $M T T$ regime $(a, b, c, d)$ a pathological scenario can be identified for low $C B F$ and prolonged $M T T$, that is the right side of images $(a, c)$ and the left side of images $(b, d)$. In this condition, DCB shows the best attachment to the ground-truth (dashed lines). Overall, DCB deconvolution performs better or at least comparably to CPI on dispersion-free data, whereas both of the techniques improve results compared to oSVD. This result is relevant for DCB: in fact, it is more general than CPI since it is designed to handle both dispersion-free and dispersed scenarios.

\subsection{Influence of dispersion on $D C B$ and $o S V D$ results}

In this section, we compare the two non-parametric approaches to deconvolution, DCB and oSVD, in the presence of increasing levels of dispersion. For this experiment we use the EDK dispersion kernel varying the vascular mean transit time $\left(M T T_{v}=\right.$ $1 / \beta)$ in range $M T T_{v} \in[1,10] s$. The ground-truth $R^{*}(t)$ was calculated with eq. (10). Signals are generated with time-step $\Delta t=0.1 \mathrm{~s}$ and then down-sampled to obtain a repetition time $T R=1 \mathrm{~s}$. The tested delay values are $0,1,2,3,4,5$ seconds (Mehndiratta et al., 2014a) but for DCB the delay is researched in a broader range $[-2,7] s$ with $\tau_{s}=1 s$. Other tested parameters are $M T T \in\{4,8,12,16\} s$ and $C B F \in\{15,30,45,60\} \mathrm{ml} / 100 \mathrm{~g} / \mathrm{min}$. The comparison is performed by calculating the mean error and standard deviation over 1000 noisy realizations. In fig. 5, the left and the central images report the relative errors for $C B F^{*}$ and $M T T^{*}$, and the right image reports the absolute error for the time of maximum $t_{\max }$ of the effective response function $R^{*}(t)$. The right image additionally reports the absolute error of the dispersion time $\tau_{\delta}$ calculated with DCB deconvolution. 

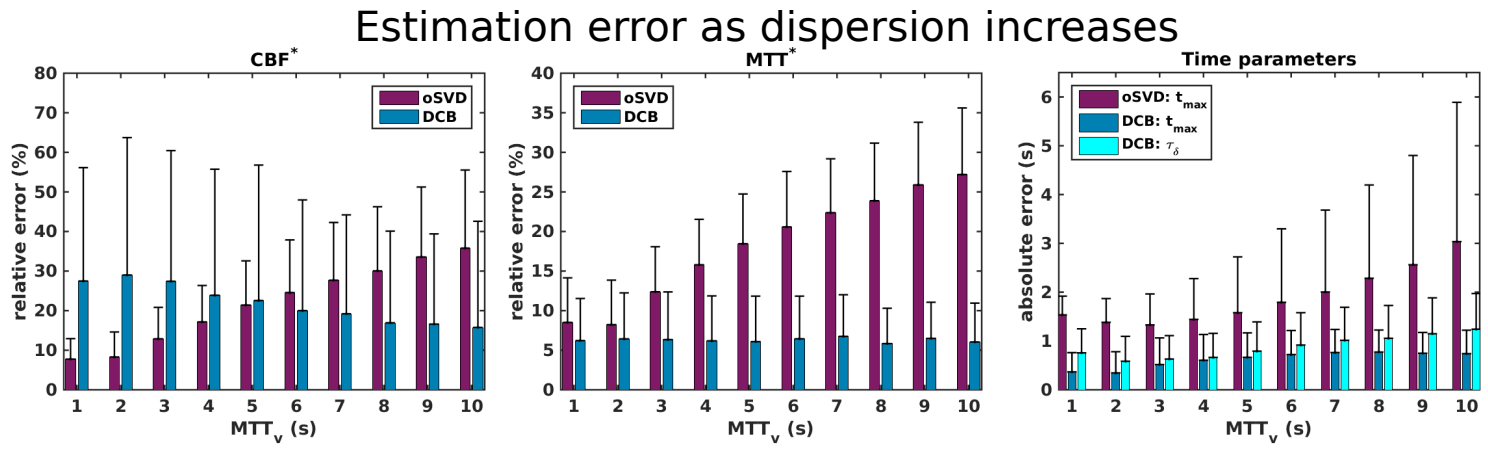

Figure 5: Estimation error (mean and standard deviation) obtained with DCB and oSVD deconvolution as the amount of dispersion increases in abscissa with the vascular mean transit time $M T T_{v}$. The figure reports the relative estimation errors of the effective perfusion parameters: $C B F^{*}$ (left) and $M T T^{*}$ (central). The barplot in the right image reports absolute errors of the time of maximum $t_{\max }$ of the effective response function and, only for DCB, the dispersion time $\tau_{\delta}$ (see fig. 2). The synthetic data was generated accounting for a dispersed response function with exponential dispersion kernel (EDK), i.e. eq. (10), varying $C B F, M T T$ and delay $\tau$ as specified in section 4.2. The DCB results remain more stable than oSVD ones as dispersion increases and show a globally improved estimation of the tested parameters.

\subsubsection{Results}

The DCB results show overall an improved stability compared to oSVD. The oSVD estimation error for all of the tested parameters in fig. 5 increases with the vascular mean transit time $M T T_{v}$, i.e. the dispersion level. On the contrary DCB performance for $C B F^{*}$ improves with $M T T_{v}$ (fig. 5, left). The results related to the time parameters in fig. 5 (right) show that average absolute error for the estimation of $t_{\max }$ falls below $1 s$ which is sensibly lower than oSVD error at any $M T T_{v}$. The image also shows the absolute error for the dispersion time $\tau_{\delta}$ obtained with DCB deconvolution. The dispersion time is a parameter derived from the joint estimation of the time of maximum $t_{\max }$ and the time delay $\tau$, therefore the error on $\tau_{\delta}$ accounts for both of the error contributions. The error on $\tau_{\delta}$ estimation is also indicative of the performance of $\mathrm{DCB}$ deconvolution in characterizing dispersion, as discussed in section 2.3. We note that the DCB mean absolute error falls approximately within $1 s$ which corresponds to the temporal resolution, i.e. the repetition time $T R$, used for the experiment. The oSVD technique scores the best performance in $C B F^{*}$ estimation at low $M T T_{v}$, likely because of its implementation as discussed in section 3.2, but renders a higher error for $M T T^{*}$.

Results globally show that deconvolution with DCB is more robust, with respect to dispersion, than with oSVD. Moreover, the results give an indication of the bias in the estimation of the effective parameters with oSVD as dispersion increases. In 
the next section, we propose a more extensive experiment, to compare all of the dispersion-compliant techniques (DCB, oSVD, and CPI+VTF), accounting for the other dispersion kernels in eq. (9), i.e. GDK and LNDK, and for the dispersion levels encoded in table 1.

\subsection{Comparison with oSVD and CPI+VTF in presence of dispersion}

In this experiment, we compare the performance of DCB, oSVD and CPI+VTF in estimating the parameters $C B F^{*}, M T T^{*}, t_{\max }, \tau, \tau_{\delta}$ and the fitting error. Groundtruth signals are generated as described in section 4.2. However, this experiment takes into account dispersion. Particularly, the data is generated taking into account all of the three dispersion kernels (GDK, EDK, and LNDK), as described in section 3.3 and illustrated in fig. 3, and the three dispersion levels (low, medium, and high) reported in table 1 . The DCB, oSVD, and CPI+VTF deconvolutions are performed for 100 different noisy realizations for each combination of $C B F, M T T$, $\tau$, dispersion kernel and dispersion level. Results in figs. 6 and 7 are presented in the form of error boxplots ${ }^{4}$.

In the first part of the experiment, we compute the estimates of $C B F^{*}, M T T^{*}$, $t_{\max }, \tau, \tau_{\delta}$ and the fitting error, when the ground-truth data is generated with all of the dispersion kernels but at different dispersion levels. Images in fig. 6 report the error boxplots for each estimated parameter and for each, when applicable, deconvolution technique adopted (oSVD, DCB, CPI+VTF). Each row from the first to the fifth corresponds to a different estimated parameter whereas the sixth row reports the reconstruction error with respect to the effective response function. The first column starting from the left of fig. 6, framed within a black rectangle, shows error boxplots obtained when considering results at all of the dispersion levels. Columns from the second to the fourth are related to low, medium and high levels of dispersion respectively.

In the second part of the experiment, we compute the estimates when the groundtruth data is generated considering all of the dispersion levels but with different dispersion kernels. Columns from the first to the third of fig. 7 show results when

\footnotetext{
${ }^{4}$ Each boxplot is a comprise of lower and upper whiskers within which all of the results not considered as outliers are contained. The bottom and top horizontal sides of the box indicate the $25^{t h}$ and $75^{t h}$ percentile of the results respectively. The central horizontal line of the box indicates the median whereas the height of the notch-like concavity in its correspondence describes the confidence region: boxes related to different techniques with non-overlapping confidence regions along the vertical axis show medians with $5 \%$ significant difference. The percentage of outliers is reported on top of the upper whisker.
} 
the underlying vascular transport function (VTF) corresponds to GDK, LNDK and EDK respectively.

\subsubsection{Results}

Deconvolution with DCB performs globally better than the compared techniques in the presence of dispersion, as shown in the first column of fig. 6. Indeed, for the tested parameters $M T T^{*}, t_{\max }, \tau$ and $\tau_{\delta}$ the median of the blue boxplot is lower than the comparing ones, with 5\% significant difference. Also the whiskers and horizontal sides ( $25^{\text {th }}$ and $75^{\text {th }}$ percentiles) generally compare favorably for DCB. An exception to the trend is the great performance of oSVD for $C B F^{*}$ estimation, noticed also in Mehndiratta et al. (2014a), which is counterbalanced by a poorer performance w.r.t. $M T T^{*}$. However, here the oscillation index for oSVD is chosen optimally (see section 3.2), which explains the result at least in part. Overall DCB scores the best results even if it generally leads to a higher percentage of outliers (number on top of each boxplot). CPI+VTF performs comparably to oSVD for the estimation of $M T T^{*}$ and outperforms it with respect to $t_{\max }$.

Results obtained with the three techniques show different trends as the dispersion level increases. The trend for the same technique also changes based on the considered parameter, i.e. each row of fig. 6. For instance, the time of maximum $t_{\max }$ estimation performance of both oSVD and CPI+VTF improves with the amount of dispersion, whereas DCB results look more stable and show lower or comparable errors. DCB shows a similar stability also for the effective mean transit time $M T T^{*}$.

Results for delay $\tau$ and dispersion time $\tau_{\delta}$ (fourth and fifth row of fig. 6) look similar as expected. Indeed, for both of the parameters the median error of DCB (blue boxplots) is lower than that of CPI+VTF (red boxplots). DCB error distribution tends to spread towards larger values as dispersion increases (see the $75^{\text {th }}$ percentile), whereas the median of CPI+VTF tends to decrease. However DCB deconvolution distinctly outperforms CPI+VTF at low and medium dispersion levels. Finally, the fitting error results (last row of fig. 6) favor DCB over CPI+VTF. However, we note that the general higher number of outliers for DCB might reveal more instability than the comparing techniques.

In the second part of the experiment, the results are illustrated separately for each adopted dispersion kernel (GDK, LNDK, and EDK) while accounting for all of the dispersion levels, as shown in fig. 7. Again, similar considerations as before apply to oSVD results, which reflect the trade-off between $C B F^{*}$ and $M T T^{*}$ estimation. With any dispersion kernel, results for effective mean transit time $M T T^{*}$ and time of maximum $t_{\max }$ show that DCB deconvolution outperforms oSVD and CPI+VTF. We note that for these parameters DCB performs better than or compa- 
Effective parameters: All Disperion KERNELS

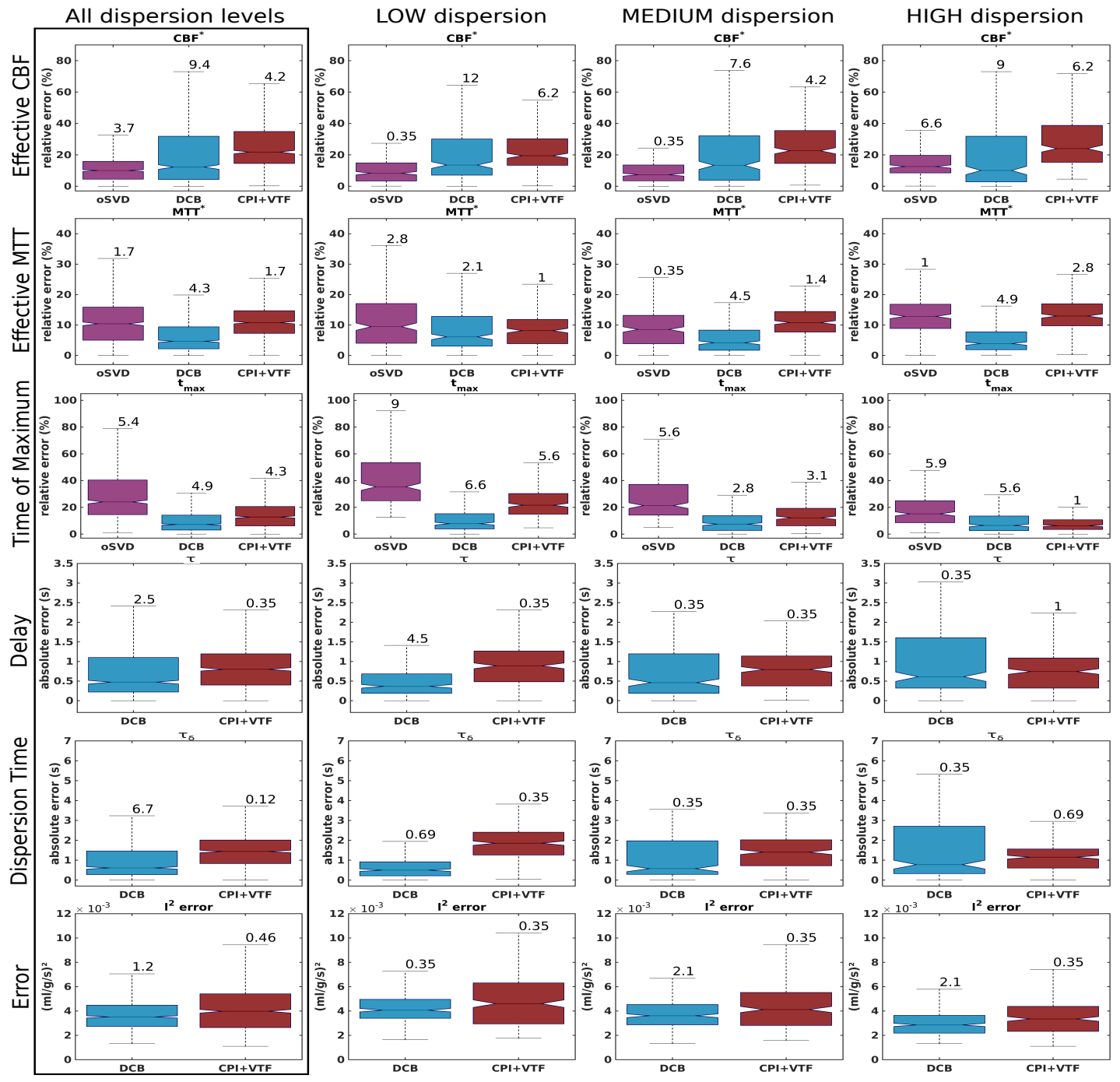

Figure 6: Error boxplots of parameters in presence of dispersion (see fig. 2 for reference) obtained with DCB (blue), oSVD (purple) and CPI+VTF (red) deconvolution. Results account for all the dispersion kernels GDK, LNDK, and EDK in eq. (9). The first framed column reports the averaged results of all the tested dispersion levels according to table 1, whereas columns from the second to the fourth report results considering separately low, medium and high dispersion. Each boxplot reports the median value (central line) with $5 \%$ confidence region (notch-like concavity), $25^{\text {th }}$ and $75^{\text {th }}$ percentile (bottom and top sides of the box), outliers-free region (between bottom and top whiskers), and outliers percentage. The data was generated varying dispersion kernel, $C B F, M T T$ and $\tau$ as specified in section 4.3. Results show lower error for DCB almost everywhere. 


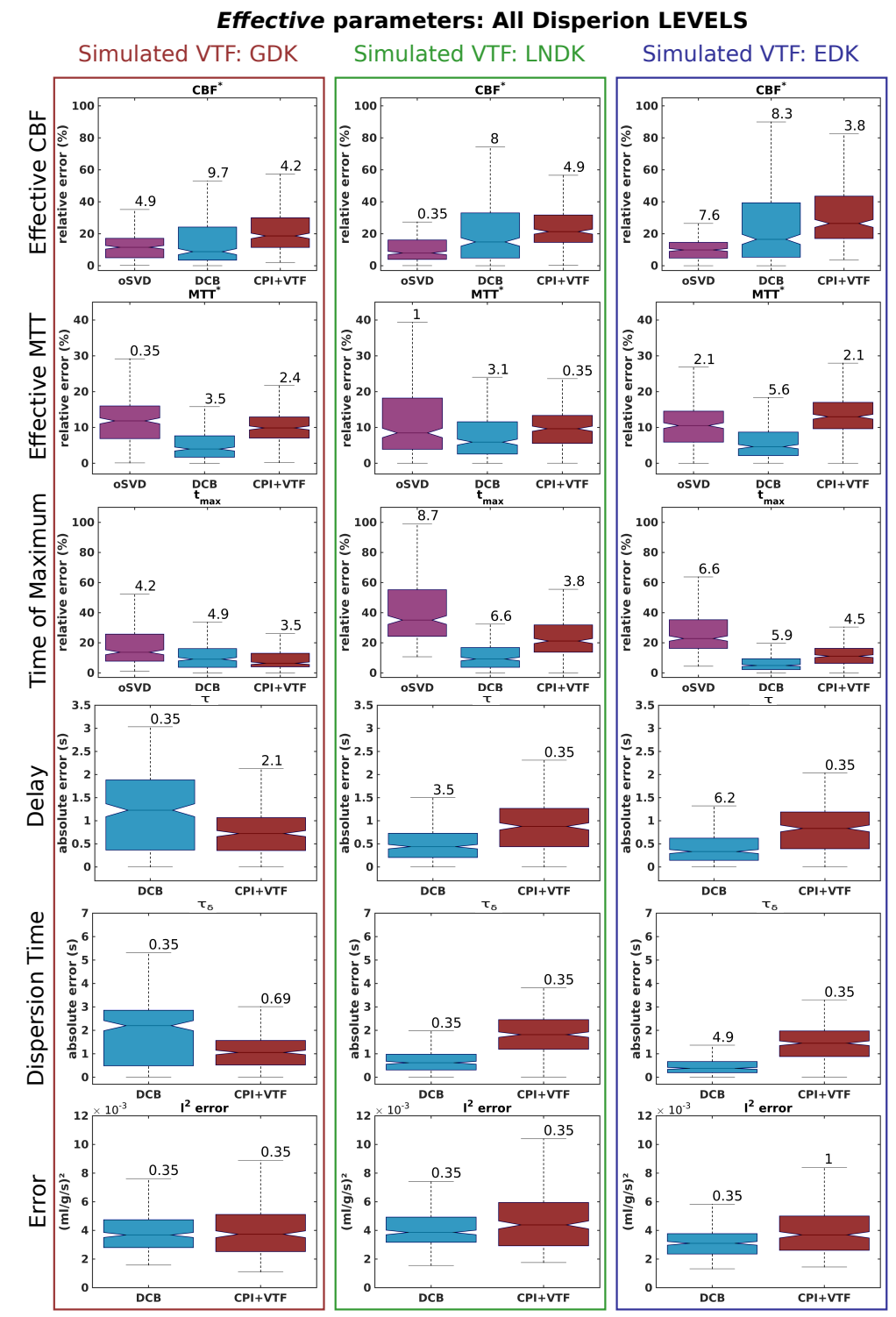

Figure 7: Error boxplots of parameters in presence of dispersion (see fig. 2 for reference) obtained with DCB (blue), oSVD (purple) and CPI+VTF (red) deconvolution. Results account for all the low, medium and high dispersion levels in table 1. Columns from the first to the third respectively report results related to dispersed data generated with the gamma (GDK), lognormal (LNDK) and exponential (EDK) dispersion kernels defined in eq. (9). Boxplots report statistics as described for fig. 6. The data was generated varying dispersion level, $C B F, M T T$ and $\tau$ as specified in section 4.3 . 
rably to $\mathrm{CPI}+\mathrm{VTF}$ also when data is generated using the gamma dispersion kernel (GDK), which is the kernel inherently assumed by the VTF model-based technique. When considering the gamma dispersion kernel (GDK), DCB deconvolution shows a performance loss in delay $\tau$ and dispersion time $\tau_{\delta}$ estimation. On the other hand DCB remarkably improves $\tau$ and $\tau_{\delta}$ estimates for data based on lognormal (LNDK) and exponential (EDK) kernels. Fitting errors generally favor DCB deconvolution over CPI+VTF with the expected exception of the GDK case, where the difference is not significant (last row of fig. 7).

Overall, the median errors reveal that DCB deconvolution performs better than $\mathrm{CPI}+\mathrm{VTF}$ and oSVD when considering all the dispersion kernels at any dispersion level (fig. 6). Results for the single dispersion kernels (fig. 7) show parameter-specific tendencies, such in the case of delay and dispersion time for the GDK, but generally confirm the positive performance of DCB deconvolution.

\subsection{DCB as pre-processing step for $C P I+V T F$}

The CPI+VTF technique, since it relies on a model of the VTF, offers the advantage of estimating the dispersion-free $C B F$ and $M T T$ directly in the deconvolution procedure. In this work, we propose instead to estimate these parameters in two steps: first, we obtain $R^{*}(t)$ via DCB deconvolution; second, we perform CPI+VTF model fitting directly on the recovered $R^{*}(t)$. We perform experiments that show the advantage of estimating $C B F$ and $M T T$ with this second method. The comparison is performed when the ground-truth data is generated accounting for all of the dispersion kernels at different dispersion levels (fig. 8), and for all the dispersion levels with different dispersion kernels fig. 9, as described in section 4.3.

\subsubsection{Results}

Results in fig. 8 show that both DCB and CPI+VTF globally render similar errors of the dispersion-free cerebral blood flow $C B F$ (first row and column). Although $\mathrm{CPI}+\mathrm{VTF}$ reduces the error at low dispersion, globally and at medium and high dispersion DCB shows the best performance. Indeed, the error with CPI+VTF increases with the level of dispersion. In addition, a previous deconvolution with DCB followed by the CPI+VTF model fitting considerably reduces the estimation error of the mean transit time $M T T$ at all the dispersion levels (globally a $50 \%$ error reduction), as shown in the first column of fig. 8. Also in this case, the amount of error with CPI+VTF increases with the dispersion level. The comparison that considers different dispersion kernels (GDK, LNDK, EDK), shown in fig. 9, brings evidence of the benefit of pre-processing data with DCB for the joint estimation of $C B F$ and $M T T$. Overall, the pre-processing with DCB allows a clear improvement in the recovery of dispersion-free parameters, for all dispersion levels and kernels. 


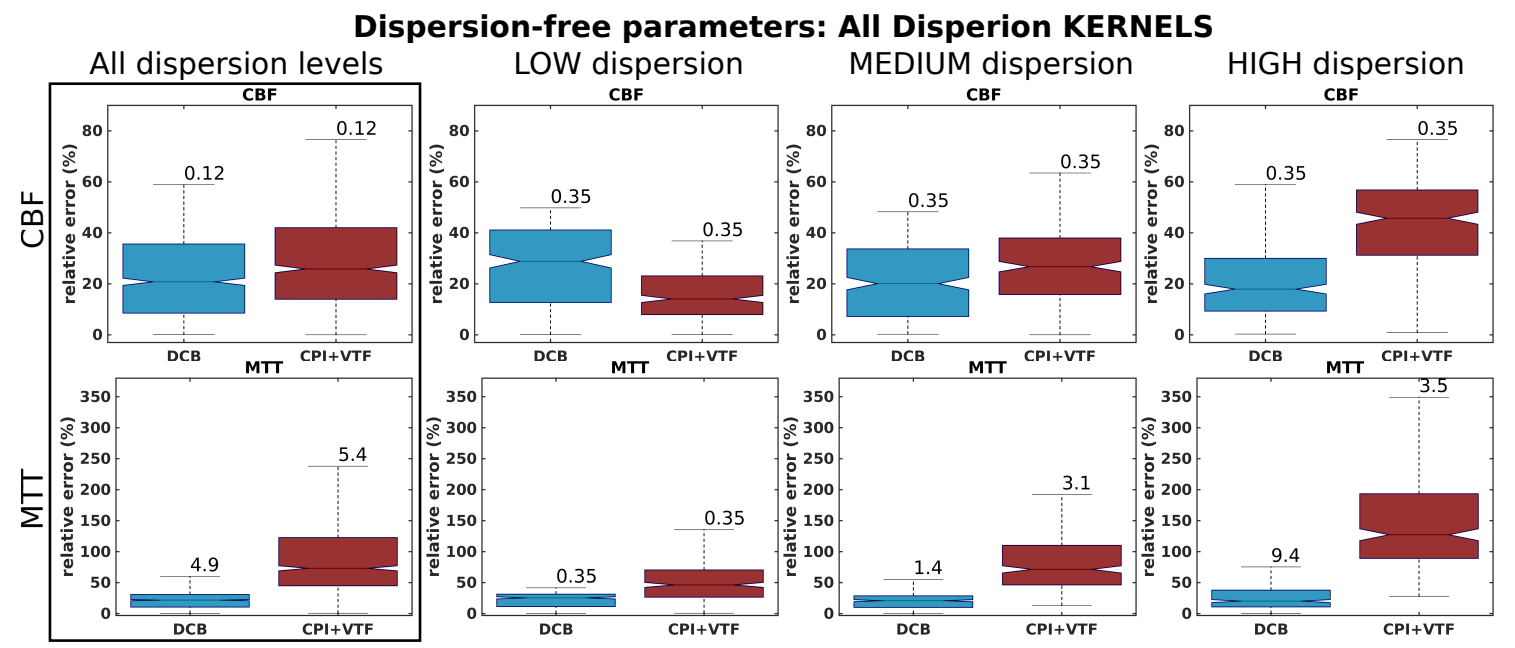

Figure 8: Error boxplots of the actual dispersion-free $C B F$ (first row) and $M T T$ (second row) parameters obtained in presence of dispersion with DCB (blue) and CPI+VTF (red). In the case of DCB, results are obtained by fitting the inherent CPI+VTF model to the effective response function previously calculated via DCB deconvolution. Results are presented considering all the dispersion kernels for different dispersion levels as in fig. 6. Pre-processing data with DCB has a beneficial effect on the joint estimation of $C B F$ and $M T T$ estimation, at any level of dispersion.

Dispersion-free parameters: All Disperion LEVELS

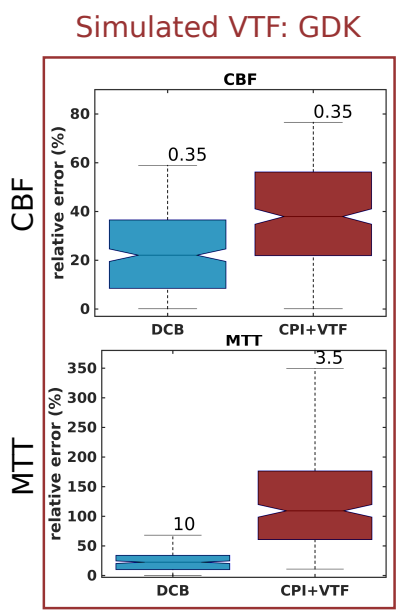

Simulated VTF: LNDK

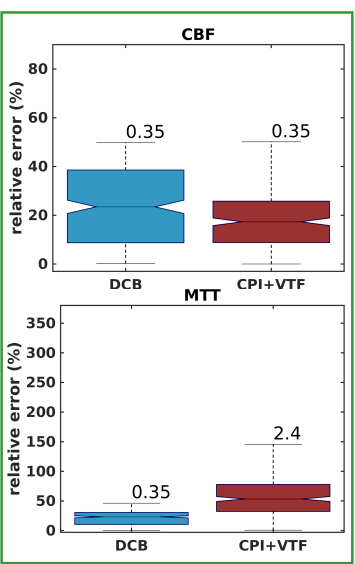

Simulated VTF: EDK

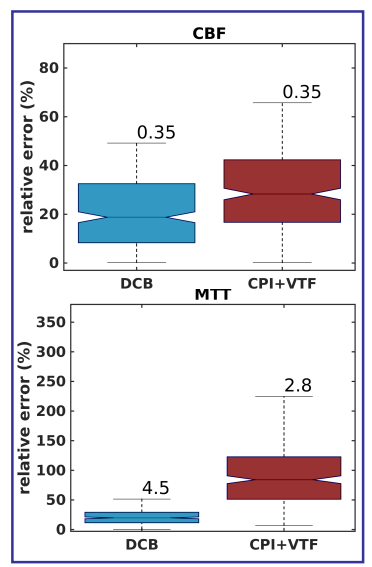

Figure 9: Error boxplots of the actual dispersion-free $C B F$ (first row) and $M T T$ (second row) parameters obtained in presence of dispersion with DCB (blue) and CPI+VTF (red). DCB results are obtained as described for fig. 8. Results are presented considering all the dispersion levels for different dispersion kernels as in fig. 7. Pre-processing data with DCB has a beneficial effect on the joint estimation of $C B F$ and $M T T$ estimation, with any dispersion kernel. 

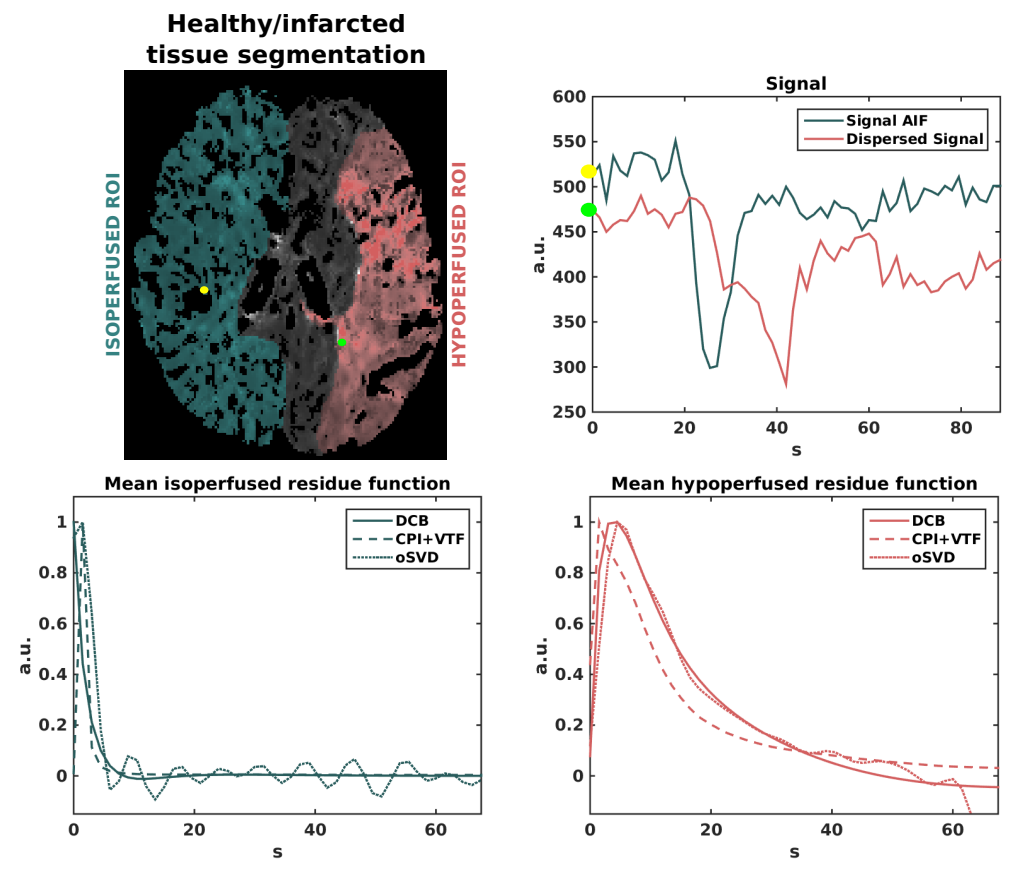

Figure 10: Top left: manual segmentation of healthy isoperfused tissue (light blue) and infarcted hypoperfused region (red) based on $M T T^{*}$ maps of fig. 12. Top right: signals corresponding to the arterial input function, manually selected in the branch of the right middle cerebral artery (see yellow dot), and to a voxel in the hypoperfused region (green dot). Bottom left and right: average tissue residue function (response function normalized by $C B F^{*}$ ), estimated with DCB (solid line), CPI+VTF (dashed line), and oSVD (dotted line) for the isoperfused region (left) and the hypoperfused one (right). The DCB render a dispersion-free shape in the healthy tissue whereas oSVD and CPI+VTF show a dispersed profile. Moreover, in the infarcted tissue DCB and oSVD show a smoother and more dispersed profile than CPI+VTF.

\section{In Vivo results}

We manually segmented a slice of the real dataset into healthy isoperfused and infarcted hypoperfused regions. The segmentation is reported in the top left corner of fig. 10. The measured $C_{a}(t)$, i.e. the arterial input function (AIF), was manually selected within the healthy region in the branch of the right middle cerebral artery. The top right image of fig. 10 shows the signals corresponding to the AIF and to a voxel in the infarcted region. In order to give an idea of data quality we also provide an estimation of the SNR. This is based on the baseline signals, i.e. before tracer arrival, and is expressed as $\mathrm{SNR}=\mathbb{E}\left[S_{0}^{a}\right] / \sigma_{0}^{t s}$, where $\mathbb{E}\left[S_{0}^{a}\right]$ is the expectation of the selected arterial baseline signal and $\sigma_{0}^{t s}$ is the calculated standard deviation of the baseline tissue signal in each voxel. Figure 11 shows the SNR map for a slice of 

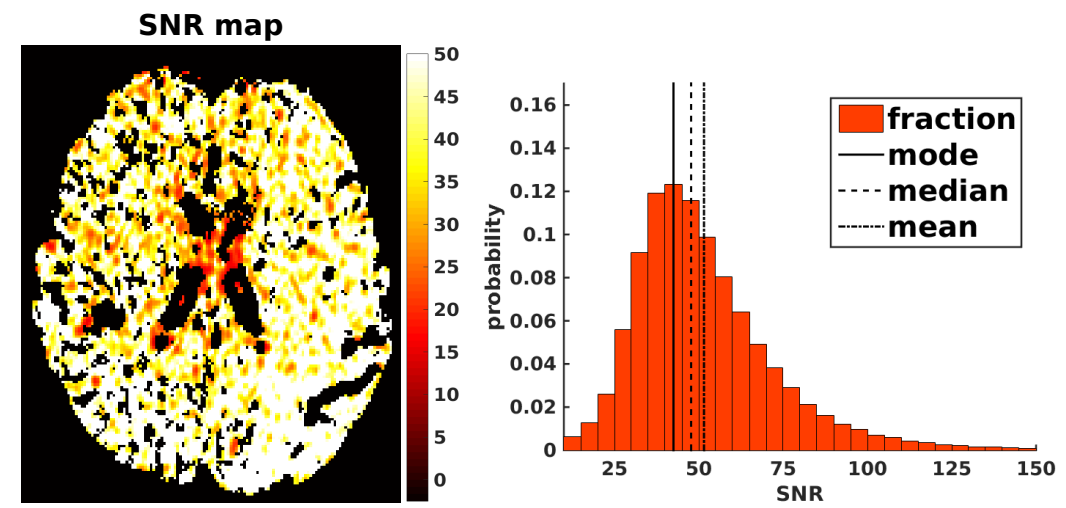

Figure 11: Estimated Signal to Noise Ratio (SNR) map of the selected slice (left), and the histogram of the SNR values for the entire acquired volume (right). The mean SNR is greater than 50 .

interest (left) and the histogram of the SNR values for the whole acquired volume (right). The mean SNR is above 50.

We estimated the response function and the perfusion parameters with oSVD, $\mathrm{DCB}$ and $\mathrm{CPI}+\mathrm{VTF}$. For DCB and $\mathrm{CPI}+\mathrm{VTF}$, results are obtained searching for the time-delay $\tau$ in range $[0,15] \mathrm{s}$, with $\tau_{s}=T R=1.5 \mathrm{~s}$. The bottom images of fig. 10 report the mean residue functions, i.e. response functions normalized by $C B F^{*}$, obtained with the three techniques for the healthy (left) and infarcted (right) regions.

Images in fig. 12 report the $C B F, C B V, M T T$ and $t_{\max }$ maps obtained with the three tested methods. Maps of delay $\tau$ and dispersion time $\tau_{\delta}$ for DCB and CPI+VTF (and absolute difference) are shown in fig. 13. In fact, these maps are not supported by oSVD deconvolution. Finally, fig. 14 reports the maps of actual dispersion-free cerebral blood flow $C B F$ and mean transit time $M T T$ obtained with $\mathrm{CPI}+\mathrm{VTF}$ model fitting on the response function obtained with DCB (first column) and with $\mathrm{CPI}+\mathrm{VTF}$ deconvolution directly (second column). The absolute difference maps are reported in the third column. In the case of DCB the model fitting is performed only on voxels where the dispersion time is not null $\left(\tau_{\delta}>0\right)$, that is only for the voxels where dispersion is detected. Indeed, in non-dispersed voxels the effective perfusion parameters are also the actual dispersion-free ones, i.e. $C B F=C B F^{*}$ and $M T T=M T T^{*}$. In figs. 12 to 14, large vessels were removed using a threshold on $C B V$ values, i.e. voxels with $C B V>3 \%$ are set to zero (Boutelier et al., 2012), whereas cerebro spinal fluid (CSF) was previously removed. No subsequent filtering or smoothing was applied.

We also report quantitative results for the iso- and hypo-perfused regions shown in the top left image of fig. 10. Particularly, we generated histograms of $C B F$, $C B F^{*}, M T T$ and $M T T^{*}$ for DCB, CPI+VTF, and oSVD when applicable. These 
are shown in fig. 15 (isoperfused) and in fig. 16 (hypoperfused).

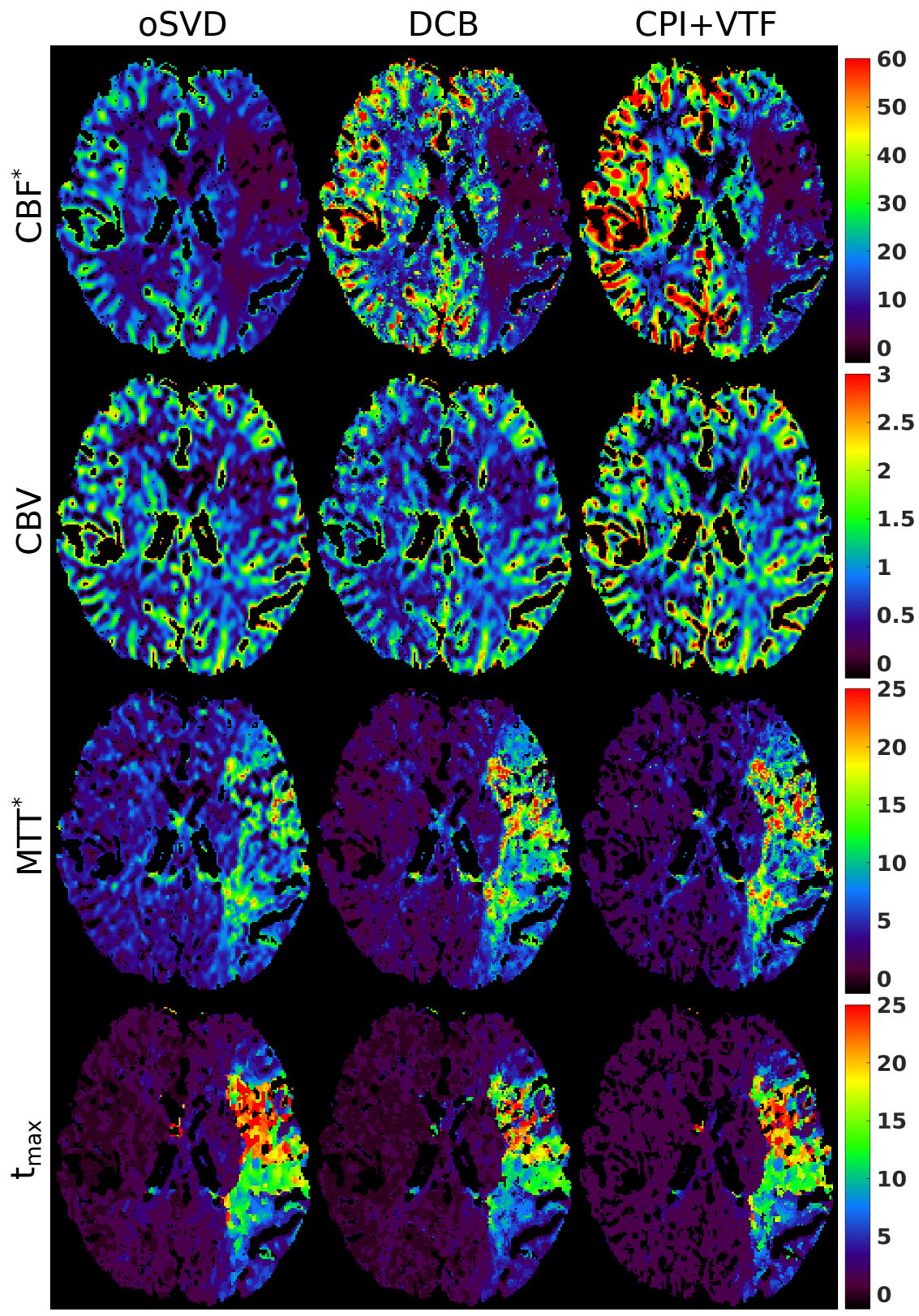

Figure 12: Maps of a $256 \times 256 \mathrm{MR}$ stroke case slice reporting the parameters $C B F^{*}(\mathrm{ml} / 100 \mathrm{~g} / \mathrm{min})$, $C B V(\%), M T T^{*}(s)$, and $t_{\max }(s)$ calculated on the effective response function $R^{*}(t)$ obtained with oSVD (first column), DCB (central column) and CPI+VTF (right column) deconvolution. Voxels with $C B V>3 \%$ were set to background to remove large vessels. 


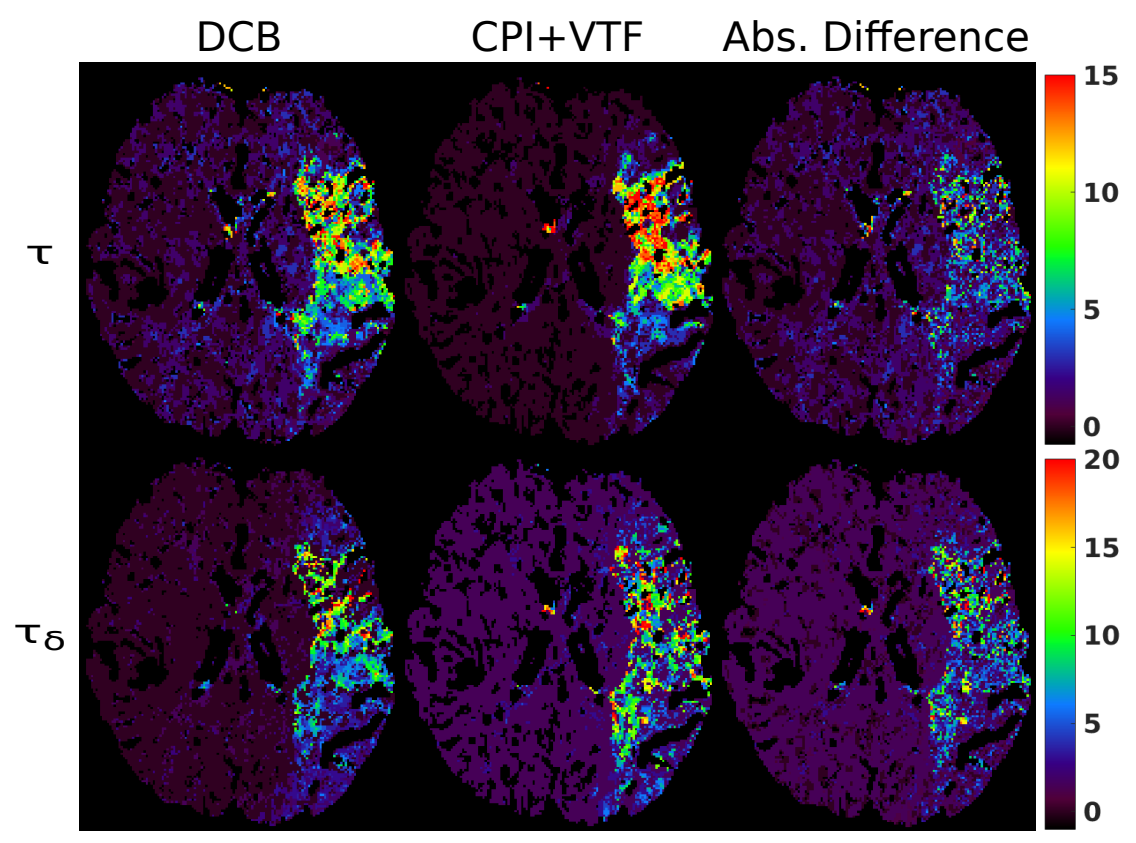

Figure 13: Maps of a $256 \times 256 \mathrm{MR}$ stroke case slice reporting delay $\tau(s)$ and dispersion time $\tau_{\delta}(s)$ calculated on the effective response function $R^{*}(t)$ obtained with DCB (left column) and CPI+VTF (central column) deconvolution. The absolute difference map is reported in the right column. As for fig. 12 voxels with $C B V>3 \%$ were set to background to remove the contribution of large vessels whereas CSF was previously removed. Contrary to CPI+VTF, the DCB map of dispersion time displays several voxels with $\tau_{\delta}=0$ implying absence of dispersion (see section 2.3). For DCB, dispersion-free voxels are mainly concentrated in the left hemisphere, contralaterally to the infarcted region.

\subsection{Results}

In general, all the techniques show decreased values of $C B F^{*}$ (first row of fig. 12) in the infarcted hypoperfused region (right side region in top left image of fig. 10) and corresponding increased $M T T^{*}$. The maps shown fig. 12 are qualitatively similar, but oSVD shows less contrast between the regions for both of the parameters. This is quantified in the histograms reported in figs. 15 and 16. The maps of dispersion time $\tau_{\delta}$ in fig. 13 show that DCB detects none or little dispersion in the healthy region, whereas CPI+VTF renders dispersed response functions almost everywhere. A more direct evidence of this is given by the shape of the mean residue functions in the bottom left image of fig. 10. On the other side, DCB renders a more dispersed response function within the infarcted region (bottom right image of fig. 10), in agreement with oSVD findings. Qualitatively, dispersion-free maps in fig. 14, generated with $\mathrm{CPI}+\mathrm{VTF}$ and DCB, report similar information. However, the DCB-based estimates 


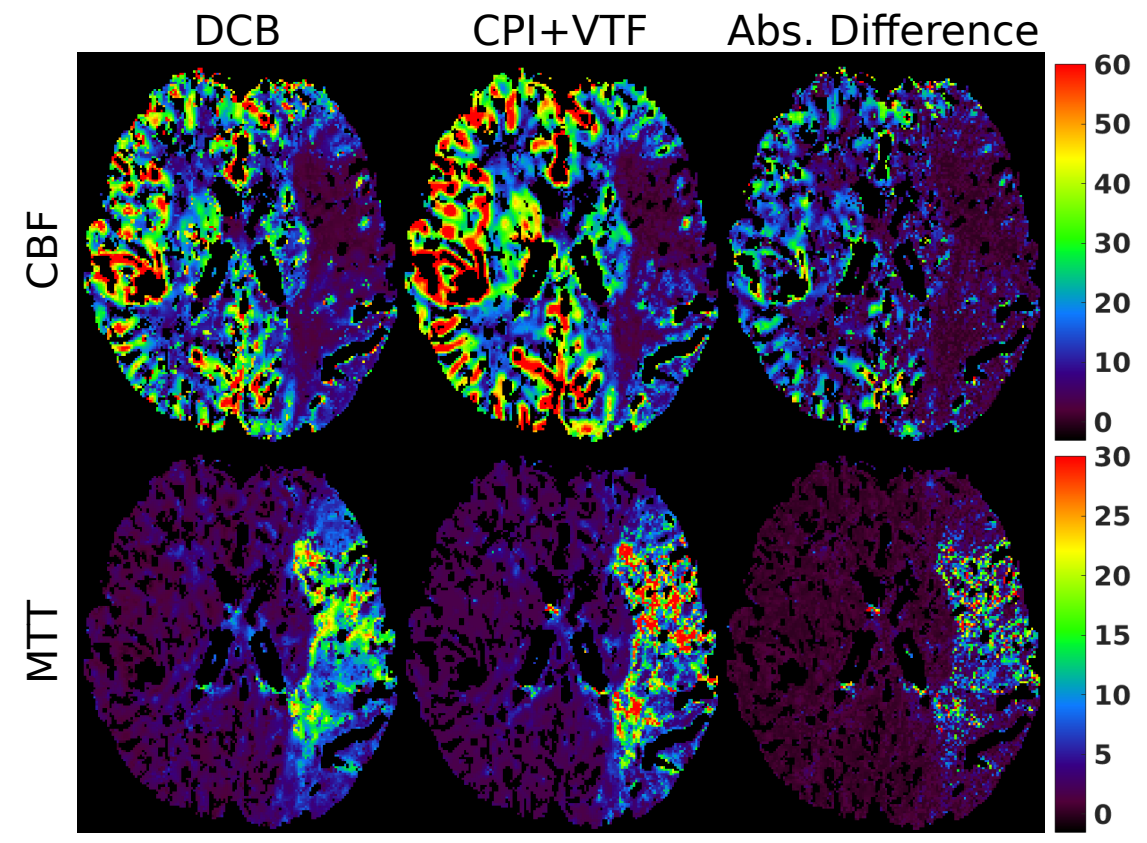

Figure 14: Maps of a $256 \times 256 \mathrm{MR}$ stroke case slice of figs. 12 and 13 reporting the actual dispersionfree $C B F(\mathrm{ml} / 100 \mathrm{~g} / \mathrm{min})$ and $M T T(s)$ obtained with DCB (left column) and CPI+VTF (central column) deconvolution. The absolute difference map is reported in the right column. The DCB maps of $C B F$ and $M T T$ report respectively the corresponding $C B F^{*}$ and $M T T^{*}$ values of fig. 12 for voxels where no dispersion has been detected in the dispersion time map of fig. 13, i.e. $\tau_{\delta}=0$. In voxels where dispersion is detected DCB results of $C B F$ and $M T T$ are obtained by fitting the $\mathrm{CPI}+\mathrm{VTF}$ inherent model to the effective response function obtained via previous deconvolution with dispersion-compliant bases (DCB), as for fig. 8.

of $M T T$ within the hypoperfused region are more compatible with a dispersion-free scenario. Indeed, after removing the bias due to dispersion, the dispersion-free $M T T$ should not be larger than the apparent effective one, i.e. $M T T \leq M T T^{*}$. In the following, we discuss results in detail.

\section{Cerebral Blood Flow}

Maps of $C B F^{*}$ obtained with oSVD look smoother but seem to be visually less discriminating than those obtained with DCB and CPI+VTF (first row of fig. 12). Indeed, a comparison between the $C B F^{*}$ histograms in the first row of fig. 15 and fig. 16 reveals that the change in width of the oSVD distribution between iso- and hypo-perfused regions is smaller than with the other techniques. In fact, both DCB and CPI+VTF show a more evident change in spread with a support extending up to $100 \mathrm{ml} / 100 \mathrm{~g} / \mathrm{min}$ in the isoperfused Region Of Interest (ROI) that reduces to 
ISOPERFUSED ROI
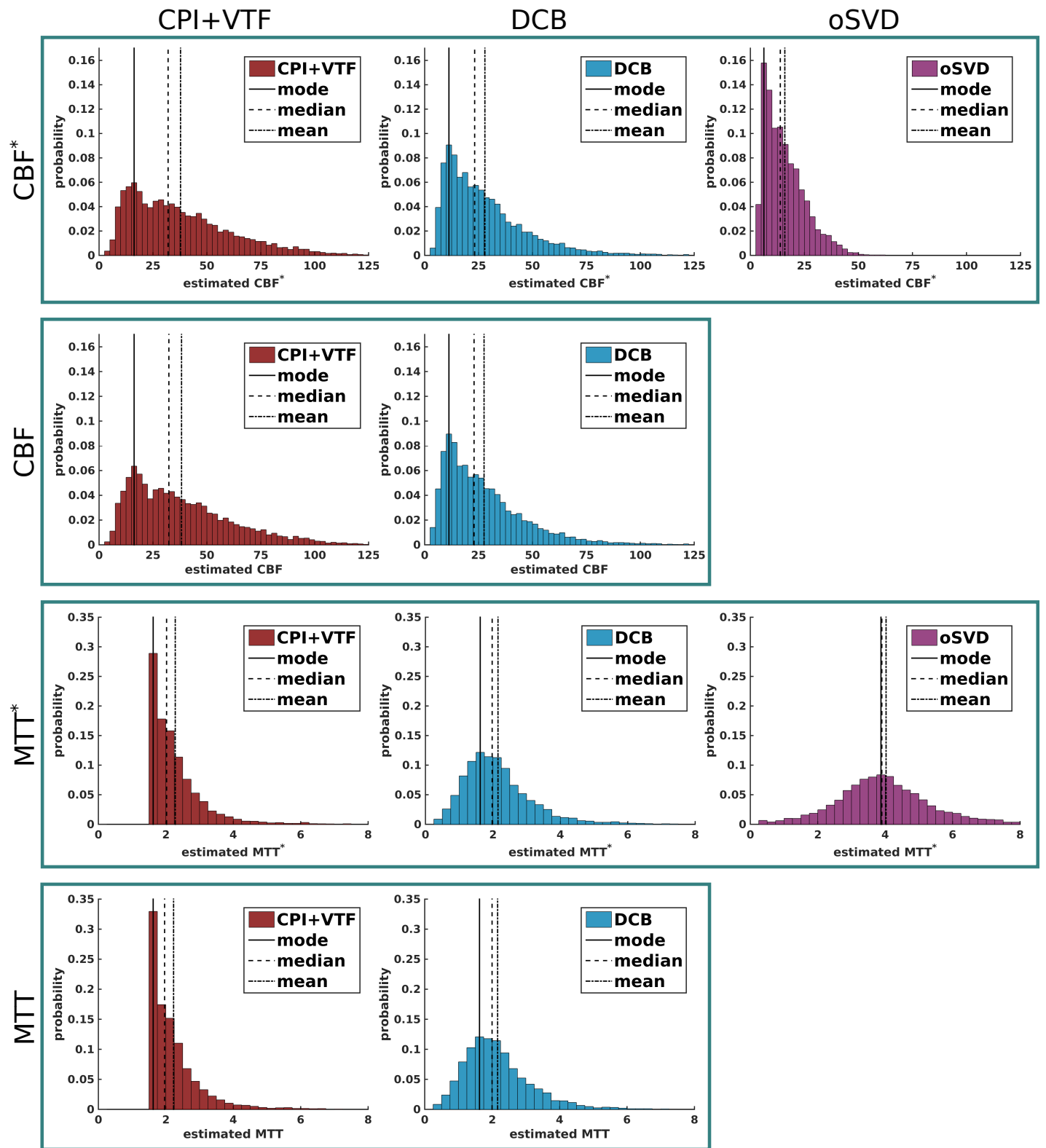

Figure 15: Histograms of $C B F^{*}, C B F, M T T^{*}$, and $M T T$ corresponding to the voxels within the isoperfused healthy ROI of fig. 10 for CPI+VTF (first column), DCB (central column) and, when applicable, oSVD (right column). Images also report the mode, mean and median values. 
HYPOPERFUSED ROI
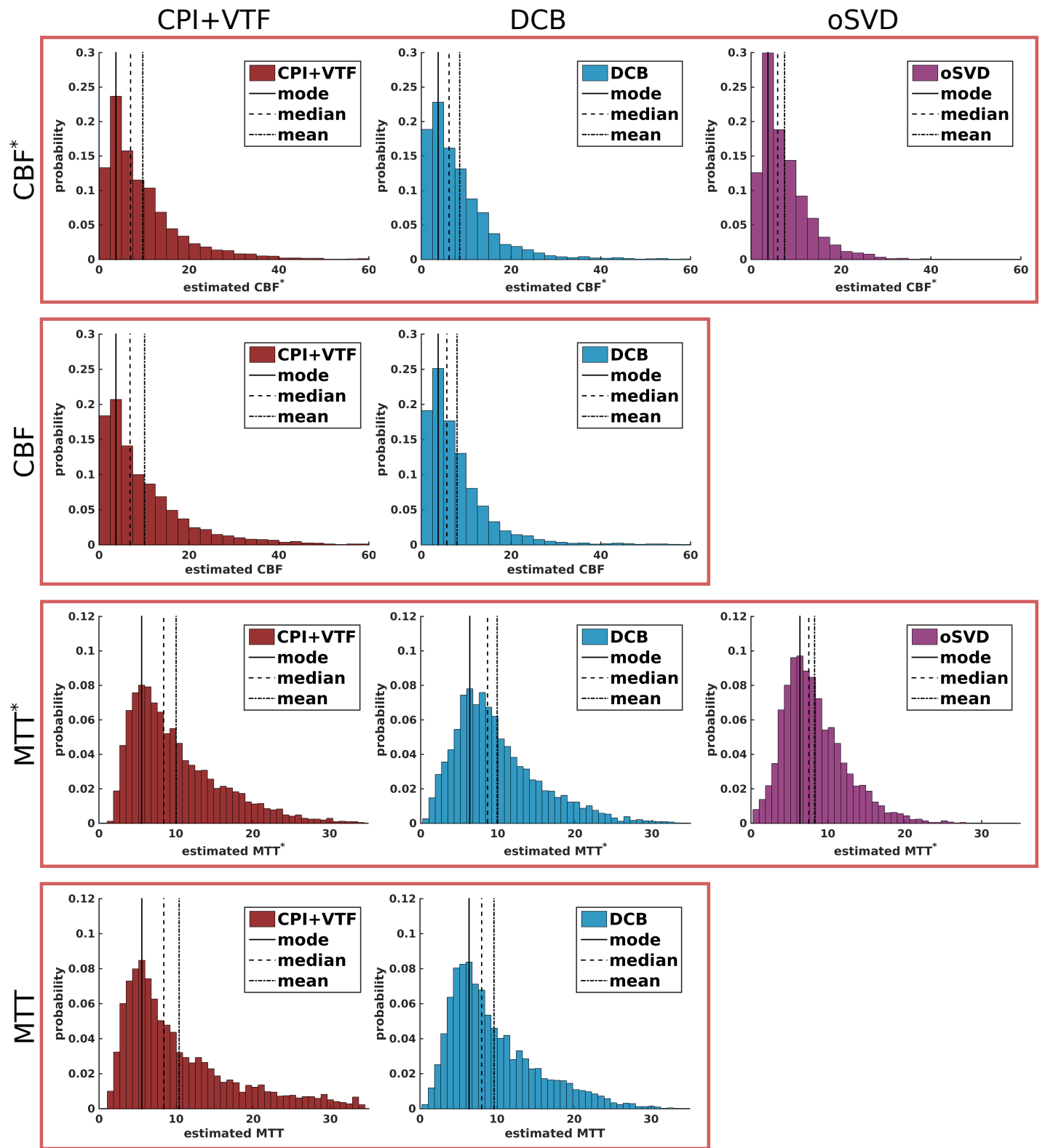

Figure 16: Histograms of $C B F^{*}, C B F, M T T^{*}$, and $M T T$ corresponding to the voxels within the hypoperfused infarcted ROI of fig. 10 for CPI+VTF (first column), DCB (central column) and, when applicable, oSVD (right column). Images also report the mode, mean and median values. 
approximately $40 \mathrm{ml} / 100 \mathrm{~g} / \mathrm{min}$ in the hypoperfused one. In the isoperfused ROI the $C B F^{*}$ histograms of the compared techniques are different from each other (fig. 15). The oSVD shows the lowest median and the smallest spread whereas CPI+VTF shows the highest median and largest spread. The histogram of DCB presents an intermediate situation. In general, $C B F^{*}$ histograms for oSVD, DCB, and CPI+VTF are in better agreement with each other in the hypoperfused ROI (fig. 16) than in the healthy isoperfused one.

\section{Mean Transit Time}

All of the methods reveal that $M T T^{*}$ increases in the hypoperfused area (third row of fig. 12) in accordance with the infarcted condition of the tissue. However, the $\mathrm{DCB}$ and $\mathrm{CPI}+\mathrm{VTF}$ maps show the greatest contrast between healthy and infarcted regions. This is supported by the $M T T^{*}$ histograms in figs. 15 and 16 (third row), where the ratio of the medians between hypo/iso-perfused regions is approximately $8.5 s / 2 s$ for DCB and CPI+VTF, and only $7.5 s / 4 s$ for oSVD. We also note that the distribution of $M T T^{*}$ for the isoperfused ROI is different among the techniques. With this regard, CPI+VTF renders estimates which have a lower bound $\left(M T T^{*}\right.$ histogram of fig. 15), whereas DCB and oSVD estimates reach lower values. Indeed, these two techniques are not constrained to render non-negative solutions of the response function, which could explain in part this phenomenon.

\section{Time of maximum, delay and dispersion time}

Maps of the time of maximum $t_{\max }$ of the effective response function, shown in the last row of fig. 12, reveal to be discriminating between the iso- and hypoperfused ROIs with all of the tested techniques. The $t_{\max }$ values obtained with DCB visually look smaller than with oSVD, which typically overestimates (see results in figs. 5, 6 and 9). In the hypoperfused region oSVD and CPI+VTF maps look visually similar whereas the DCB map shows a reduced amount of high estimates (red areas). In the isoperfused region both oSVD and DCB show voxels where $t_{\max }=0$ whereas $\mathrm{CPI}+\mathrm{VTF}$ almost never renders such a value. This is reflected also in the dispersion time $\tau_{\delta}$ maps of fig. 13 (second row), where CPI+VTF finds $\tau_{\delta}>0$ almost everywhere. This phenomenon may constitute a bias for CPI+VTF because it implies that every voxel in the healthy region presents an effective response function with a dispersed shape. The mean residue function recovered with CPI+VTF, shown in the bottom left image of fig. 10, confirms this "dispersion-detecting" trend. Indeed, DCB renders a dispersion-free shape in the healthy tissue whereas CPI+VTF shows a dispersed profile. It is interesting to note that in the infarcted area DCB shows a smoother and more dispersed profile, which is in better agreement with oSVD than with CPI+VTF (bottom right image). We note that oSVD solution, when 
the response function is smooth as in the case of high dispersion, is a good solution since the high-frequency cutoff likely removes only noise and not discontinuities, as opposed to what would happen in the case of an exponential response function.

The delay $\tau$ map for CPI+VTF in fig. 13 also depicts no variability within the isoperfused region, whereas DCB presents positive delays more frequently. In addition, DCB seems to render lower delays in the hypoperfused ROI, which has to be ascribed to corresponding lower values of $t_{\max }$. In the isoperfused region, DCB seems to split the time of maximum contributions more on the delay than on the dispersion time compared to CPI+VTF. Globally, oSVD seems to overestimate $t_{\text {max }}$. To summarize, DCB renders in average a dispersion-free response function profile within the healthy region, as opposed to oSVD and CPI+VTF, and a smooth dispersed profile within the infarcted one.

\section{Dispersion-free parameters}

The maps of the actual dispersion-free perfusion parameters in fig. 14 obtained with DCB and CPI+VTF show similar contrast between healthy and infarcted ROIs, but present substantial differences. For instance, we note that the CPI+VTF map of $C B F$ reports higher values than the corresponding DCB ones in the isoperfused region (see segmentation in fig. 10). This is confirmed in fig. 15: $C B F$ histogram of DCB shows a higher density for values lower than $50 \mathrm{ml} / 100 \mathrm{~g} / \mathrm{min}$, whereas with $\mathrm{CPI}+\mathrm{VTF}$ the histogram spreads and presents a higher density of values above $75 \mathrm{ml} / 100 \mathrm{~g} / \mathrm{min}$. On the other hand, CBF histograms (DCB and CPI+VTF) in the hypoperfused region bear similar information (fig. 16). The MTT maps in fig. 14 of DCB and CPI+VTF present differences particularly in the hypoperfused region, where the DCB map shows lower values. To clarify, in the presence of dispersion detected by both the techniques as shown in the second row of fig. 13 - the dispersionfree $M T T$ could be expected to be lower than the corresponding effective $M T T^{*}$, due to the underestimation of the cerebral blood flow. However, with CPI+VTF, the value of $M T T$ in the dispersion-positive hypoperfused region is higher than the corresponding $M T T^{*}$. Indeed, in the passage from the $M T T^{*}$ to MTT the histogram of CPI+VTF (third and fourth rows of fig. 16) we see an increased density of values above $20 \mathrm{~s}$. On the other hand, the median and mean values of the DCB histogram sensibly decrease. To summarize, DCB pre-processing renders lower $C B F$ estimates within the healthy region, where it detects no dispersion, and lower $M T T$ estimates within the infarcted region, where dispersion is detected in agreement with pathological conditions. 


\section{Discussion}

In DSC-MRI, data can generally contain voxels with no dispersion, dispersed voxels within healthy tissue (Østergaard et al., 1998; Calamante et al., 2000), and voxels where dispersion is the result of a pathological scenario such as a steno-occlusive disease (Calamante et al., 2003b). Dispersion is related to macrovascular phenomena and constitutes a confounding factor for the measurement of the microvascular response function and tissue perfusion.

In this work, we developed a deconvolution technique, based on DispersionCompliant Bases (DCB), that handles dispersed data without, at the same time, making assumptions about the macrovascular process, i.e. it does not adopt a model of the vascular transport function. The DCB method is in this sense "dispersion insensitive", i.e. it allows recovering the effective response function when data is dispersed or dispersion-free. Interestingly, DCB performed comparably better than oSVD and CPI in the dispersion-free experiments depicted in fig. 4, and globally rendered a higher accuracy than oSVD and CPI+VTF in the presence of dispersion, as shown in figs. 6 and 7. In addition, DCB pre-processing helped the separation of micro- and macrovascular effects in silico, i.e. it reduced the error of the dispersionfree $C B F$ and $M T T$ estimates obtained via CPI+VTF model fitting, as shown in figs. 8 and 9. We note that in some synthetic experiments DCB renders a higher number of outliers, which is probably due to less stability in the low SNR regime compared to oSVD (which was performed with an optimal oscillation index as described in section 3.2) and CPI+VTF. However we think that integrating DCB deconvolution in a denoising framework that accounts for neighborhood information, such as the total variation method in Fang et al. (2015), could help improving stability.

We believe that dispersion should be taken into account when performing DSCMRI deconvolution. Indeed, dispersion can induce broadening of the effective response function, as shown in fig. 2, and neglecting it could lead to overestimation of MTT and underestimation of CBF (Calamante et al., 2000). To this regard, we propose to use the dispersion time $\tau_{\delta}$ for dispersion detection and characterization. This, in practice, can only be achieved with DCB and CPI+VTF, since oSVD does not allow estimating time-delay, $\tau$, and time of maximum, $t_{\text {max }}$, which are required for the calculation of the dispersion time. However, synthetic results in figs. 6 and 7 suggest that DCB deconvolution globally renders better estimates of $\tau_{\delta}$. Also in real data, DCB deconvolution managed to correctly recover voxels with zero dispersion time, which implies voxels where no dispersion occurs. These non-dispersed voxels are mainly concentrated in the healthy isoperfused region, where CPI+VTF renders $\tau_{\delta}>0$ almost everywhere instead. Consequently, all the voxels processed with $\mathrm{CPI}+\mathrm{VTF}$ have to be interpreted as dispersed, as demonstrated by the correspond- 
ing shape of the mean residue function in the bottom left image of fig. 10. This might be the cause of the higher $C B F$ values, observed within the healthy region, when compared to DCB deconvolution (maps in fig. 14 and histograms in fig. 15). In fact, the pre-processing with DCB deconvolution offers the advantage of selecting the voxels affected by dispersion - when $\tau_{\delta}>0$ - so that we can avoid fitting a VTF model based technique (such as CPI+VTF or others) in dispersion-free voxels, thus reducing the risk of overfitting. Conceptually, if the reported DCB dispersion time map in fig. 13 is correct, as suggested by the synthetic experiments in fig. 6, it means that $\mathrm{CPI}+\mathrm{VTF}$ detects dispersed shapes where there is no dispersion, which might lead to a consequent overestimation of the dispersion-free $C B F$ (see the absolute difference map in fig. 14). To this regard, in this work CPI+VTF was not implemented with the original bayesian variational method as reported in Chappell et al. (2009), and this constitutes a limitation of our study. Indeed, we did not implement the a priori information elaborated in Mehndiratta et al. (2014a), which is designed to bias the estimation of the effective response function towards a dispersed shape with high probability for low-to-medium level of dispersion. However, we think that such a bias could emphasize the "dispersion-detecting" behavior of CPI+VTF in the healthy region.

The computational cost of DCB and CPI+VTF is higher than oSVD. However for a comparable number of parameters adopted, DCB is significantly faster than $\mathrm{CPI}+\mathrm{VTF}$ thanks to its analytic Jacobian. The atomic cost per iteration for each voxel is $0.014 s$ for oSVD, $0.67 s$ for DCB and $0.83 s$ for CPI+VTF on an Intel $\left(\right.$ Core $^{\mathrm{TM}}$ i7-3840QM 2.80GHz CPU, with 32GB RAM. However, the techniques are suitable for code optimization and parallel computing.

The proposed Dispersion-Compliant Bases method (DCB) allows us to explain data without assumptions about the presence, amount, or nature of dispersion. The method reconstructs plausible response functions (fig. 10) in healthy and pathological scenarios. Moreover, it globally renders better estimates of the effective perfusion parameters, and its use as a pre-processing step boosts the precision of dispersion-free estimates of $C B F$ and $M T T$.

\section{Conclusion}

In this work, we describe a deconvolution method based on Dispersion-Compliant Bases which is capable of detecting the presence of dispersion and recovering the corresponding effective response function. The method proves to be accurate in both dispersion-free and dispersed scenarios. It improves the estimation of the effective perfusion parameters in the presence of dispersion and reduces the error of the 
dispersion-free mean transit time and cerebral blood flow estimates. We believe that the presented bases approach to DSC-MRI deconvolution brings added value to the state-of-the-art and better explains the data, which is a fundamental step before inferring any model-related property.

\section{Acknowledgments}

The author Marco Pizzolato expresses his thanks to Olea Medical and to the Provence-Alpes-Côte d'Azur Regional Council for providing grant and support.

\section{References}

Axel, L., 1980. Cerebral blood flow determination by rapid-sequence computed tomography: theoretical analysis. Radiology 137, 679-686.

Bassingthwaighte, J., Goresky, G., 1984. The cardiovascular system., in: Handbook of Physiology. Springer, pp. 549-626.

Batchelor, P., Chiribiri, A., Nooralipour, N.Z., Cvetkovic, Z., 2010. ARMA regularization of cardiac perfusion modeling., in: ICASSP, pp. 642-645.

Bjørnerud, A., Emblem, K.E., 2010. A fully automated method for quantitative cerebral hemodynamic analysis using DSC-MRI. Journal of Cerebral Blood Flow \& Metabolism 30, 1066-1078.

Boutelier, T., Kudo, K., Pautot, F., Sasaki, M., 2012. Bayesian hemodynamic parameter estimation by bolus tracking perfusion weighted imaging. Medical Imaging, IEEE Transactions on 31, 1381-1395.

Calamante, F., 2005. Bolus dispersion issues related to the quantification of perfusion MRI data. Journal of magnetic resonance imaging 22, 718-722.

Calamante, F., 2013. Arterial input function in perfusion MRI: a comprehensive review. Progress in nuclear magnetic resonance spectroscopy 74, 1-32.

Calamante, F., Gadian, D., Connelly, A., 2002. Quantification of Perfusion Using Bolus Tracking Magnetic Resonance Imaging in Stroke: Assumptions, Limitations, and Potential Implications for Clinical Use. Stroke 33, 1146-1151.

Calamante, F., Gadian, D.G., Connelly, A., 2000. Delay and Dispersion Effects in Dynamic Susceptibility Contrast MRI Simulations Using Singular Value Decomposition . 
Calamante, F., Gadian, D.G., Connelly, A., 2003a. Quantification of bolus-tracking MRI: Improved characterization of the tissue residue function using Tikhonov regularization. Magnetic Resonance in Medicine 50, 1237-1247.

Calamante, F., Thomas, D.L., Pell, G.S., Wiersma, J., Turner, R., 1999. Measuring cerebral blood flow using magnetic resonance imaging techniques. Journal of cerebral blood flow \& metabolism 19, 701-735.

Calamante, F., Willats, L., Gadian, D.G., Connelly, A., 2006. Bolus delay and dispersion in perfusion MRI: implications for tissue predictor models in stroke. Magnetic Resonance in Medicine 55, 1180-1185.

Calamante, F., Yim, P.J., Cebral, J.R., 2003b. Estimation of bolus dispersion effects in perfusion MRI using image-based computational fluid dynamics. NeuroImage 19, 341-353.

Chappell, M., Groves, A., Whitcher, B., Woolrich, M., 2009. Variational Bayesian Inference for a Nonlinear Forward Model. IEEE Transactions on Signal Processing $57,223-236$.

Chappell, M.A., Woolrich, M.W., Kazan, S., Jezzard, P., Payne, S.J., MacIntosh, B.J., 2013. Modeling dispersion in arterial spin labeling: validation using dynamic angiographic measurements. Magnetic Resonance in Medicine 69, 563-570.

Charter, M., Gull, S., 1987. Maximum entropy and its application to the calculation of drug absorption rates. Journal of pharmacokinetics and biopharmaceutics 15 , 645-655.

Connelly, A., Calamante, F., Willats, L., 2006. Improved deconvolution of bolus tracking data using wavelet thresholding, in: Proc. 14th Sci. Meeting Int. Soc. Magn. Reson. Med., pp. 3563-3563.

Drabycz, S.A., Brown, R.A., Law, A.G., Mitchell, J.R., 2005. Maximum entropy deconvolution for dynamic susceptibility contrast magnetic resonance imaging, in: Visualization, Imaging, And Image Processing: Fifth IASTED International Conference Proceedings.

Fang, R., Zhang, S., Chen, T., Sanelli, P.C., 2015. Robust low-dose ct perfusion deconvolution via tensor total-variation regularization. IEEE transactions on medical imaging 34, 1533-1548. 
Hamilton, W.F., Moore, J.W., Kinsman, J., Spurling, R., 1932. Studies on the circulation. American Journal of Physiology-Legacy Content 99, 534-551.

Jacquez, J.A., 1972. Compartmental analysis in biology and medicine .

Keeling, S.L., Kogler, T., Stollberger, R., 2009. Deconvolution for DCE-MRI using an exponential approximation basis. Medical Image Analysis 13, 80-90.

Kiselev, V.G., 2005. Transverse relaxation effect of MRI contrast agents: A crucial issue for quantitative measurements of cerebral perfusion. Journal of Magnetic Resonance Imaging 22, 693-696.

Kiselev, V.G., Posse, S., 1999. Analytical model of susceptibility-induced MR signal dephasing: effect of diffusion in a microvascular network. Magnetic resonance in medicine 41, 499-509.

Knutsson, L., Ståhlberg, F., Wirestam, R., 2010. Absolute quantification of perfusion using dynamic susceptibility contrast MRI: pitfalls and possibilities. Magnetic Resonance Materials in Physics, Biology and Medicine 23, 1-21.

Ko, L., Salluzzi, M., Frayne, R., Smith, M., 2007. Reexamining the quantification of perfusion MRI data in the presence of bolus dispersion. Journal of Magnetic Resonance Imaging 25, 639-643.

Lassen, N.A., Andersen, A.R., Friberg, L., Paulson, O.B., 1988. The retention of [99mtc]-d, l-HM-PAO in the human brain after intracarotid bolus injection: a kinetic analysis. J Cereb Blood Flow Metab 8, S13-S22.

Mehndiratta, A., Calamante, F., MacIntosh, B.J., Crane, D.E., Payne, S.J., Chappell, M.A., 2014a. Modeling and correction of bolus dispersion effects in dynamic susceptibility contrast MRI: Dispersion Correction with CPI in DSC-MRI. Magnetic Resonance in Medicine 72, 1762-1774.

Mehndiratta, A., Calamante, F., MacIntosh, B.J., Crane, D.E., Payne, S.J., Chappell, M.A., 2014b. Modeling the residue function in DSC-MRI simulations: Analytical approximation to in vivo data: Modeling Residue Function for DSC-MRI. Magnetic Resonance in Medicine 72, 1486-1491.

Mehndiratta, A., MacIntosh, B.J., Crane, D.E., Payne, S.J., Chappell, M.A., 2013. A control point interpolation method for the non-parametric quantification of cerebral haemodynamics from dynamic susceptibility contrast MRI. NeuroImage 64, $560-570$. 
Meier, P., Zierler, K.L., 1954. On the theory of the indicator-dilution method for measurement of blood flow and volume. Journal of applied physiology 6, 731-744.

Meijs, M., Christensen, S., Lansberg, M.G., Albers, G.W., Calamante, F., 2015. Analysis of perfusion mri in stroke: To deconvolve, or not to deconvolve. Magnetic resonance in medicine .

Mouannes-Srour, J.J., Shin, W., Ansari, S.A., Hurley, M.C., Vakil, P., Bendok, B.R., Lee, J.L., Derdeyn, C.P., Carroll, T.J., 2012. Correction for arterial-tissue delay and dispersion in absolute quantitative cerebral perfusion DSC MR imaging. Magnetic Resonance in Medicine 68, 495-506.

Mouridsen, K., Friston, K., Hjort, N., Gyldensted, L., Østergaard, L., Kiebel, S., 2006. Bayesian estimation of cerebral perfusion using a physiological model of microvasculature. Neuroimage 33, 570-579.

Østergaard, L., Chesler, D.A., Weisskoff, R.M., Sorensen, A.G., Rosen, B.R., 1999. Modeling cerebral blood flow and flow heterogeneity from magnetic resonance residue data. Journal of Cerebral Blood Flow \& Metabolism 19, 690-699.

Østergaard, L., Johannsen, P., Høst-Poulsen, P., Vestergaard-Poulsen, P., Asboe, H., Gee, A.D., Hansen, S.B., Cold, G.E., Gjedde, A., Gyldensted, C., 1998. Cerebral blood flow measurements by magnetic resonance imaging bolus tracking: comparison with [15o] h2o positron emission tomography in humans. Journal of Cerebral Blood Flow \& Metabolism 18, 935-940.

Østergaard, L., Weisskoff, R.M., Chesler, D.A., Gyldensted, C., Rosen, B.R., 1996. High resolution measurement of cerebral blood flow using intravascular tracer bolus passages. part i: Mathematical approach and statistical analysis. Magnetic resonance in medicine $36,715-725$.

Park, C.S., Payne, S.J., 2013. A generalized mathematical framework for estimating the residue function for arbitrary vascular networks. Interface focus 3, 20120078.

Rempp, K.A., Brix, G., Wenz, F., Becker, C.R., Gückel, F., Lorenz, W.J., 1994. Quantification of regional cerebral blood flow and volume with dynamic susceptibility contrast-enhanced mr imaging. Radiology 193, 637-641.

Rohrer, M., Bauer, H., Mintorovitch, J., Requardt, M., Weinmann, H.J., 2005. Comparison of magnetic properties of mri contrast media solutions at different magnetic field strengths. Investigative radiology 40, 715-724. 
Rosen, B.R., Belliveau, J.W., Buchbinder, B.R., McKinstry, R.C., Porkka, L.M., Kennedy, D.N., Neuder, M.S., Fisel, C.R., Aronen, H.J., Kwong, K.K., et al., 1991. Contrast agents and cerebral hemodynamics. Magnetic resonance in medicine 19, 285-292.

Rosen, B.R., Belliveau, J.W., Vevea, J.M., Brady, T.J., 1990. Perfusion imaging with nmr contrast agents. Magnetic resonance in medicine 14, 249-265.

Shiroishi, M.S., Castellazzi, G., Boxerman, J.L., D’Amore, F., Essig, M., Nguyen, T.B., Provenzale, J.M., Enterline, D.S., Anzalone, N., Drfler, A., Rovira, ., Wintermark, M., Law, M., 2015. Principles of $\mathrm{T}_{2}{ }^{*}$-weighted dynamic susceptibility contrast MRI technique in brain tumor imaging: Principles of $\mathrm{T}_{2}$ *-Weighted DSC-MRI. Journal of Magnetic Resonance Imaging 41, 296-313.

Smith, M., Lu, H., Trochet, S., Frayne, R., 2004. Removing the effect of svd algorithmic artifacts present in quantitative $\mathrm{mr}$ perfusion studies. Magnetic resonance in medicine 51, 631-634.

Speck, O., Chang, L., DeSilva, N.M., Ernst, T., 2000. Perfusion MRI of the human brain with dynamic susceptibility contrast: Gradient-echo versus spin-echo techniques. Journal of Magnetic Resonance Imaging 12, 381-387.

Starmer, C., Clark, D.O., 1970. Computer computations of cardiac output using the gamma function. Journal of applied physiology 28, 219-220.

Stewart, G.N., 1894. Researches on the circulation time in organs and on the influences which affect it. The Journal of physiology 15, 1-45.

Villringer, A., Rosen, B.R., Belliveau, J.W., Ackerman, J.L., Lauffer, R.B., Buxton, R.B., Chao, Y.S., Wedeenand, V.J., Brady, T.J., 1988. Dynamic imaging with lanthanide chelates in normal brain: contrast due to magnetic susceptibility effects. Magnetic resonance in medicine 6, 164-174.

Vonken, E.j.P.A., Beekman, F.J., Bakker, C.J., Viergever, M.A., 1999a. Maximum likelihood estimation of cerebral blood flow in dynamic susceptibility contrast MRI. Magnetic resonance in Medicine 41, 343-350.

Vonken, E.j.P.A., van Osch, M.J., Bakker, C.J., Viergever, M.A., 1999b. Measurement of cerebral perfusion with dual-echo multi-slice quantitative dynamic susceptibility contrast MRI. Journal of Magnetic Resonance Imaging 10, 109-117. 
Weisskoff, R., Zuo, C.S., Boxerman, J.L., Rosen, B.R., 1994. Microscopic susceptibility variation and transverse relaxation: theory and experiment. Magnetic Resonance in Medicine 31, 601-610.

Willats, L., Connelly, A., Calamante, F., 2006. Improved deconvolution of perfusion MRI data in the presence of bolus delay and dispersion. Magnetic Resonance in Medicine 56, 146-156.

Willats, L., Connelly, A., Calamante, F., 2007. Modelling the bolus dispersion from DSC-MRI data, in: Proceedings of the International Society for Magnetic Resonance in Medicine (ISMRM), 15th Annual Meeting, Berlin, Germany, pp. 14451445.

Willats, L., Connelly, A., Calamante, F., 2008. Minimising the effects of bolus dispersion in bolus-tracking MRI. NMR in Biomedicine 21, 1126-1137.

Willats, L., Connelly, A., Christensen, S., Donnan, G.A., Davis, S.M., Calamante, F., 2012. The role of bolus delay and dispersion in predictor models for stroke. Stroke 43, 1025-1031.

Wirestam, R., Ståhlberg, F., 2005. Wavelet-based noise reduction for improved deconvolution of time-series data in dynamic susceptibility-contrast MRI. Magnetic Resonance Materials in Physics, Biology and Medicine 18, 113-118.

Wu, O., Østergaard, L., Weisskoff, R.M., Benner, T., Rosen, B.R., Sorensen, A.G., 2003. Tracer arrival timing-insensitive technique for estimating flow in $\mathrm{mr}$ perfusion-weighted imaging using singular value decomposition with a blockcirculant deconvolution matrix. Magnetic resonance in medicine 50, 164-174.

Zanderigo, F., Bertoldo, A., Pillonetto, G., Cobelli, C., 2009. Nonlinear Stochastic Regularization to Characterize Tissue Residue Function in Bolus-Tracking MRI: Assessment and Comparison With SVD, Block-Circulant SVD, and Tikhonov. IEEE Transactions on Biomedical Engineering 56, 1287-1297.

Zierler, K.L., 1962. Theoretical basis of indicator-dilution methods for measuring flow and volume. Circulation Research 10, 393-407.

Zierler, K.L., 1965. Equations for measuring blood flow by external monitoring of radioisotopes. Circulation Research 16, 309-321. 